\title{
SUPERBOX - An Efficient Code for Collisionless Galactic Dynamics
}

\author{
M. Fellhauer ${ }^{a, 1}$ P. Kroupa ${ }^{b, 2}$ H. Baumgardt ${ }^{c, 3}$ R. Bien ${ }^{a, 1}$ \\ C.M. Boily ${ }^{\mathrm{a}, 1}$ R. Spurzem ${ }^{\mathrm{a}, 1}$ N. Wassmer ${ }^{\mathrm{d}}$ \\ ${ }^{a}$ Astronomisches Rechen-Institut, Mönchhofstr. 12-14, D-69120 Heidelberg, Germany \\ ${ }^{\mathrm{b}}$ Institut für Theoretische Astrophysik, Universität Heidelberg, Tiergartenstr. 15, \\ D-69121 Heidelberg, Germany \\ ${ }^{\mathrm{c}}$ Department of Mathematics and Statistics, University of Edinburgh, King's Buildings, \\ Edinburgh EH9 3JZ, Scotland (UK) \\ ${ }^{\mathrm{d}}$ Robert Bosch GmbH, Robert Bosch Str. 1, D-77815 Buehl, Germany
}

\begin{abstract}
We present SUPERBOX, a particle-mesh code with high resolution sub-grids and an NGP (nearest grid point) force-calculation scheme based on the second derivatives of the potential. SUPERBOX implements a fast low-storage FFT-algorithm, giving the possibility to work with millions of particles on desk-top computers. Test calculations show energy and angular momentum conservation to one part in $10^{5}$ per crossing-time. The effects of grid and numerical relaxation remain negligible, even when these calculations cover a Hubbletime of evolution. As the sub-grids follow the trajectories of individual galaxies, the code allows a highly resolved treatment of interactions in clusters of galaxies, such as highvelocity encounters between elliptical galaxies and the tidal disruption of dwarf galaxies. Excellent agreement is obtained in a comparison with a direct-summation N-body code running on special-purpose GRAPE 3 hardware. The orbital decay of satellite galaxies due to dynamical friction obtained with SUPERBOX agrees with Chandrasekhar's treatment when the Coulomb logarithm $\ln \Lambda \approx 1.5$.
\end{abstract}

Key words: methods: numerical - galaxies: evolution - galaxies: interactions - galaxies: kinematics and dynamics

1 e-mail: mike,reinhold,cmb,spurzem@ari.uni-heidelberg.de

2 e-mail: pavel@ita.uni-heidelberg.de

3 e-mail: holger@maths.ed.ac.uk 


\section{Introduction}

In direct-summation N-body methods, the computational time $t_{C P U}$ scales with the square of the particle number $N_{\mathrm{p}}, t_{C P U} \sim N_{\mathrm{p}}^{2}$. This relation inevitably leads to bottlenecks when computing large-N galaxy models. While it is still impossible to model galaxies star by star over sensible time-laps, alternative algorithms have been developed over a number of years which ease the computer requirements at the expense of the exact $N_{\mathrm{p}}^{2}$ summation, yet provide an adequate treatment of Newtonian galactic dynamics. Examples of such techniques are the tree method (Barnes \& Hut 1986, Davé, Dubinski \& Hernquist 1997), and the multi-grid or nested grid particle mesh-codes like PANDORA (Villumsen 1989), HydRA (Pearce \& Couchman 1997), SUPERBOX, or combinations of the methods like the adaptive refinement tree (ART) (Kravtsov, Klypin \& Khokhlov. 1997), which can cope with very inhomogeneous matter distributions and have high spatial resolution at the density maxima.

While the hierarchical tree-method is independent of the geometry of the problem, its main disadvantage is the limitation in particle number and the softening needed to avoid undesired two-body relaxation. In contrast, the main advantage of a particle-mesh technique is the ability to use very high particle numbers (up to several millions on desk-top computers), because the CPU-time scales only linearly with the number of particles, and with $N_{\mathrm{gc}} \log N_{\mathrm{gc}}$, where $N_{\mathrm{gc}}$ is the number of grid-cells. High particle numbers keep the statistical noise low. The disadvantage of a grid-based method, however, is the dependency on the geometry of the grid. But with increasing spatial resolution, the geometry of the cells becomes less important. The only limitation in resolving phenomena is the size of one grid-cell. A general discussion and comparison of various numerical schemes will be found in Sellwood (1987), and - in the context of direct $N$-body simulations - in Spurzem (1999).

The basic idea behind SUPERBOX, as originally conceived by R. Bien (Bien et al. 1991), is to increase the resolution only at places where it is necessary, while simultaneously keeping the computational overhead as small as possible by using a fixed (i.e. not adaptive) nested grid-architecture. Accuracy is improved by the use of nested high-resolution sub-grids and a linear force interpolation to the exact position of the particle inside a cell. Two higher-resolution sub-grids are introduced: the medium-resolution grid contains an entire galaxy, and the high-resolution grid treats its core. Both grids stay focused on the galaxy, moving through the 'local universe' that is contained in the coarse outermost grid.

SUPERBOX has already been used successfully in a variety of investigations, namely in the study of the high velocity encounter of the two similar early-type galaxies NGC 4782/4783 by Madejski \& Bien (1993), in the survey of encounters between elliptical galaxies by Wassmer et al. (1993), and in the research on tidal disruption 
of satellite dwarf galaxies by Kroupa (1997) and Klessen \& Kroupa (1998). A version of SUPERBOX exists that includes sticky particles as a simple model of the dynamics of cold molecular gas clouds (Fellhauer 1996).

In this article we provide an implementation and benchmarks for the SUPERBOX algorithm. In Section 2, we lay out the mathematics behind the algorithm and give details of how the potential is mapped on the different grids. We then move on to test the code: Section 3 deals with the conservation of energy and angular momentum, while Section 4 discusses relaxation. Section 5 contains profiling information about storage and CPU-time requirements. Comparison with a direct-summation $N_{\mathrm{p}}$-body code is made in Section 6. In Section 7 we show - as an example the good agreement of satellite decay with Chandrasekhar's formula for dynamical friction, and close with conclusions in Section 8.

\section{Method}

Detailed discussions of the particle-mesh (PM) technique can be found in Eastwood \& Brownrigg (1978), Hockney \& Eastwood (1981) and Sellwood (1987).

Briefly, the array of mass densities, $\varrho_{i j k}$, is derived by counting the number of particles in each Cartesian grid cell $i, j, k$, i.e. by using the simple particle-in-cell (NGP $=$ "Nearest Grid Point") algorithm. All particles belonging to one galaxy $N_{\text {p,gal }}$ have the same mass, $m=M_{\text {gal }} / N_{\text {p,gal }}$. An alternative would be to use a cloud-incell technique, which is known to improve momentum and energy conservation, in which the mass of the particle is distributed proportionally among the neighbouring cells. This is, however, not necessary, since the number of particles is large.

Poisson's equation is solved for this density array to get the grid-based potential, $\Phi_{i j k}$, which becomes,

$$
\Phi_{i j k}=G \sum_{a, b, c=0}^{N-1} \varrho_{a b c} \cdot H_{a-i, b-j, c-k}, \quad i, j, k=0, \ldots, N-1
$$

where $N$ denotes the number of grid-cells per dimension $\left(N^{3}=N_{\mathrm{gc}}\right)$, and $H_{i j k}$ is some Green's function. To avoid this $N_{\mathrm{gc}}^{2}$ procedure, the discrete Fast Fourier Transform (FFT) is used, for which $N=2^{K}, K>0$ being an integer. The stationary Green's function is Fourier transformed once at the beginning of the calculation, and only the density array is transformed at each time-step:

$$
\hat{\varrho}_{a b c}=\sum_{i, j, k=0}^{N-1} \varrho_{i j k} \cdot \exp \left(-\sqrt{-1} \frac{2 \pi}{N}(a i+b j+c k)\right),
$$




$$
\hat{H}_{a b c}=\sum_{i, j, k=0}^{N-1} H_{i j k} \cdot \exp \left(-\sqrt{-1} \frac{2 \pi}{N}(a i+b j+c k)\right) .
$$

The two resulting arrays are multiplied cell by cell and transformed back to get the grid-based potential,

$$
\Phi_{i j k}=\frac{G}{N^{3}} \sum_{a, b, c=0}^{N-1} \hat{\varrho}_{a b c} \cdot \hat{H}_{a b c} \cdot \exp \left(\sqrt{-1} \frac{2 \pi}{N}(a i+b j+c k)\right) .
$$

The potential is differentiated numerically to estimate the accelerations for each particle, each of which is pushed forward on its orbit using the leapfrog scheme (see Fig. 1 and sections below).

\begin{tabular}{|c|}
\hline \multicolumn{2}{|c|}{ read model data } \\
\hline FFT of Green's function \\
\hline start time-step loop \\
\hline start galaxy loop \\
\hline calculate mass densities of all 5 grids \\
\hline calculate potentials (FFT) \\
\hline force calculation, update velocities \\
\hline end galaxy loop \\
\hline update positions, output data \\
\hline end time-step loop \\
\hline write final model data for restart \\
\hline
\end{tabular}

Fig. 1. Float-chart for SUPERBOX. 


\subsection{Green's function and the FFT}

The simplest Green's function is adopted, in which the side of a grid cell has unit length:

$$
\begin{aligned}
& H_{i j k}=\frac{1}{\sqrt{i^{2}+j^{2}+k^{2}}}, \quad i, j, k=0,1, . ., N \\
& H_{000}=4 / 3 .
\end{aligned}
$$

The value of $H_{000}$ is somewhat arbitrary, and $H_{000}=H_{100}$ is often used (see e.g. Sellwood 1987). Numerical tests performed with SUPERBOX show that for high particle numbers, $H_{000}=4 / 3$ yields a slightly slower drift in total energy with time (see also Section 3) than $H_{000}=1$ or 1.5 , while for low particle numbers (compared to the number of grid-cells) $H_{000}=1$ is the better choice. The reason for this is that for high particle numbers the collective force dominates over the particle-particle (pairwise) force. On the other hand, for low particle numbers (only one or a few particles are together in the same cell) pairwise forces are more dominant, and it is better to exclude 'self-gravity' (i.e. a particle feels its own force inside its cell), with $H_{000}=1$. This is consistent with analytical arguments by D. Pfenniger (private communication). He shows that in one dimension, $H_{0}=\ln 4$ minimises the power at the grid wave-number $n / 2$ for the kernel (compare with Eq. 3)

$$
\begin{aligned}
& H_{i}=1 /|i|, \quad 0<|i| \leq n / 2, \\
& H_{i}=\ln 4, \quad i=0 .
\end{aligned}
$$

He finds that the power is exactly cancelled at the wavenumber $n / 2$, and for finite $n$ can also be cancelled for a value of $H_{0}$ close to $\ln 4$. For $H_{0}>\ln 4$ the power at high frequencies increases, while for $H_{0}<\ln 4$ some wavenumbers $<n / 2$ are filtered out. In this sense, $H_{0}=\ln 4 \approx 4 / 3$ optimises the kernel. However, the 3D-problem is slightly different since forces instead of the potential are used. The artificial wavenumbers induced by the grid, that are to be minimised, are then much more numerous. This problem would need further addressing.

The FFT-algorithm gives the exact solution of the grid-based potential for a periodical system. For the exact solution of an isolated system, which is what we are interested in, the size of the density-array has to be doubled $(2 N)$, filling all inactive grid cells with zero density, and extending the Green's function in the empty regions in the following way:

$$
\begin{aligned}
H_{2 n-i, j, k} & =H_{2 n-i, 2 n-j, k}=H_{2 n-i, j, 2 n-k}=H_{2 n-i, 2 n-j, 2 n-k} \\
& =H_{i, 2 n-j, k}=H_{i, 2 n-j, 2 n-k}=H_{i, j, 2 n-k}=H_{i, j, k} .
\end{aligned}
$$

This provides the isolated solution of the potential in the simulated area between $i, j, k=0$ and $N-1$. In the inactive part the results are unphysical. To keep 
storage as low as possible, only a $2 N \times 2 N \times N$-array is used for transforming the densities, and a $(N+1) \times(N+1) \times(N+1)$-array is used for the Green's function. For a detailed discussion see Eastwood \& Brownrigg (1978) and also Hockney \& Eastwood (1981).

The FFT-routine incorporated in SUPERBOX is a simple one-dimensional FFT and is taken from Werner \& Schabach (1979) and Press et al. (1986). It is fast and makes the code portable and not machine specific. The low-storage algorithm to extend the FFT to 3 dimensions, to obtain the 3-D-potential, is taken from Hohl (1970). The performance of SUPERBOX can be increased by incorporating machine-optimised FFT routines.

\subsection{Acceleration and orbit integration}

In this section we explain the force calculation performed in SUPERBOX, and compare it with standard methods. We follow closely the notation of Hockney \& Eastwood (1981) and Couchman (1999) to document the differences.

With the mass density as an example we show, in a formal way, how its values on a 3D mesh are obtained. Using the $\delta$-functional for the particle shape function, a particle distribution $n(\mathbf{x})$ is given by

$$
n(\mathbf{x})=\sum_{\alpha} \delta^{3}\left(\mathbf{x}-\mathbf{x}_{\alpha}\right)
$$

where $\alpha$ is a summation index running over all particles, and $\mathbf{x}_{\alpha}=\left(x_{\alpha}, y_{\alpha}, z_{\alpha}\right)$ is the position vector of particle $\alpha$. As smoothing kernel we select here

$$
W(\mathbf{x}, \Delta \mathbf{x})=\Pi\left(\frac{x}{\Delta x}\right) \cdot \Pi\left(\frac{y}{\Delta y}\right) \cdot \Pi\left(\frac{z}{\Delta z}\right) .
$$

$\Delta \mathrm{x}$ denotes a vector of smoothing lengths $(\Delta x, \Delta y, \Delta z)$ in three coordinate directions. $W$ is a three dimensional generalisation of the standard top-hat function

$$
\Pi(\xi)= \begin{cases}0, & |\xi|>\frac{1}{2}, \\ \frac{1}{2}, & |\xi|=\frac{1}{2}, \\ 1, & |\xi|<\frac{1}{2} .\end{cases}
$$

Hence the smoothed mass-density field is

$$
\varrho(\mathbf{x})=m \cdot W \star n=m \cdot \int W\left(\mathbf{x}-\mathbf{x}^{\prime}, \mathbf{\Delta} \mathbf{x}\right) n\left(\mathbf{x}^{\prime}\right) \mathrm{d}^{3} \mathbf{x}^{\prime}
$$


where $m$ denotes the particle mass, and the $\star$-operator a convolution as defined by the equation above. Mathematically we get from $\varrho(\mathbf{x})$ a so-called mesh sampled functional $\varrho^{\dagger}(\mathbf{x})$ on a three dimensional mesh by

$$
\varrho^{\dagger}(\mathbf{x})=\amalg(\mathbf{x}) \cdot \varrho(\mathbf{x}),
$$

using the three-dimensional comb or sampling function defined as

$$
\amalg(\mathbf{x})=\sum_{i, j, k=0}^{N} \delta\left(x-x_{i j k}\right) \cdot \delta\left(y-y_{i j k}\right) \cdot \delta\left(z-z_{i j k}\right),
$$

where $i, j, k$ are indices of grid cells, whose centres are located at the vector points $\mathbf{x}_{i j k}=\left\{x_{i j k}, y_{i j k}, z_{i j k}\right\}$. From the functional $\varrho^{\dagger}(\mathbf{x})$ a finite set of discrete meshsampled density values $\varrho_{\mathrm{m}}^{\dagger}=\left\{\varrho_{i j k}, i, j, k=0,1, \ldots, N\right\}$ is obtained, which are defined at the centres of the grid cells. For each cell centre or mesh point they are computed by integrating $\varrho^{\dagger}$ over an arbitrarily shaped and sized volume containing just this and only this mesh point. The standard discrete FFT procedure (see above) is applied to the set of numbers $\varrho_{\mathrm{m}}^{\dagger}$ in order to determine the grid-based potential $\Phi_{\mathrm{m}}^{\dagger}=\left\{\Phi_{i j k}, i, j, k=0,1, \ldots, N\right\} . \Phi_{\mathrm{m}}^{\dagger}$ is a set of numbers resulting from the FFT and represents the mesh sampled functional $\Phi^{\dagger}(\mathbf{x})=\amalg(\mathbf{x}) \Phi(\mathbf{x})$, where $\Phi(\mathbf{x})$ is a true smooth gravitational potential. The set of values $\Phi_{\mathrm{m}}^{\dagger}$ is obtained from $\Phi^{\dagger}$ by integrating over volumes containing single mesh points, just in the same way as explained above for $\varrho$. We define the one-dimensional two-point difference operator for $x$ direction in the standard way (Hockney \& Eastwood 1981, p. 163),

$$
D_{x}(x, y, z, \Delta x)=\frac{1}{2 \Delta x}(\delta(x+\Delta x)-\delta(x-\Delta x)) \delta(y) \delta(z) .
$$

To keep the notation clearer we remain for the moment in one dimension. A first order difference approximation to the $\mathrm{x}$-component of the acceleration vector is given by

$$
a_{x}{ }^{(1)}(x, y, z, \Delta x)=D_{x} \star \Phi(x)=\frac{\Phi(x+\Delta x, y, z)-\Phi(x-\Delta x, y, z)}{2 \Delta x} .
$$

Sampling this on the mesh yields

$$
\begin{aligned}
a_{x}^{\dagger(1)}(x, y, z, \Delta x) & =\amalg(\mathbf{x}) \cdot a_{x}^{(1)}(x, y, z) \\
& =\amalg(\mathbf{x}) \cdot \frac{\Phi(x+\Delta x, y, z)-\Phi(x-\Delta x, y, z)}{2 \Delta x} .
\end{aligned}
$$

If this is integrated over volumes containing a single mesh point, again exactly as described above for the cases of $\varrho$ and $\Phi$, a set of accelerations $a_{i j k}{ }^{(1)}$ defined at the mesh point centres results: 


$$
a_{x, i j k}^{(1)}=\frac{\Phi_{i+1, j k}-\Phi_{i-1, j k}}{2 \Delta x}
$$

here we have assumed that $2 \Delta x=x_{i+1, j k}-x_{i-1, j k}$ is selected so as to match with the mesh point distances. In our notation the set of values $\left\{a_{x, i j k}{ }^{(1)}, i, j, k=\right.$ $0,1, \ldots, N\}$ is denoted by $a_{\mathrm{m}, \mathrm{x}}^{\dagger}{ }^{(1)}$. The corresponding vector components for the acceleration in $y$ and $z$ direction are analogous. In the standard particle-mesh schemes, the accelerations between the mesh centres are obtained by using the same smoothing kernel as for the density, i.e.

$$
\begin{array}{r}
a_{x}^{(1)}(x, y, z, \Delta x)=W \star a_{x}^{\dagger}(1)(x, y, z)=a_{x, i j k}{ }^{(1)} \quad \text { for } \quad x-x_{i j k}<\frac{\Delta x}{2} \\
\text { and } y-y_{i j k}<\frac{\Delta y}{2} \\
\text { and } z-z_{i j k}<\frac{\Delta z}{2} .
\end{array}
$$

The use of the $\delta$-functional for the particle shape and the top-hat function for smoothing gives the rather crude standard NGP scheme, where the acceleration on a particle is constant in each cell and has a discontinuity at the cell boundaries. A possible improvement regarding energy and momentum conservation is to use a so-called cloud-in-cell or CIC scheme, where the shape function of particles is a top-hat function, and the assignment function is a triangle function (see Hockney $\&$ Eastwood 1981). However, as one can also see in the cited book, such schemes suffer from force anisotropies, despite having very good conservation properties (i.e. the magnitude and direction of the force error depends on whether one goes parallel to the mesh coordinates or not).

Therefore a non-standard scheme is applied in SUPERBOX. It is NGP in nature, provides a very good energy and angular momentum conservation and a significant improvement for force anisotropies. Note that the rather involved mathematical formalism above will later yield significant hindsight what is special with SUPERBOX. The notation follows closely Hockney \& Eastwood's and Couchman's.

We begin with a Taylor expansion of the acceleration (for example the $x$-component) centred on $(x, y, z)$, the position of the centre of cell $i, j, k$ :

$$
\begin{aligned}
a_{x}^{(2)}(x+d x, y+d y, z+d z) & =a_{x}^{(2)}(x, y, z)+ \\
\frac{\partial a_{x}}{\partial x}(x, y, z) \cdot d x & +\frac{\partial a_{x}}{\partial y}(x, y, z) \cdot d y+\frac{\partial a_{x}}{\partial z}(x, y, z) \cdot d z \\
& +\mathcal{O}\left(\mathbf{d x}^{2}\right)
\end{aligned}
$$

where $\mathcal{O}\left(\mathbf{d x}^{2}\right)$ denotes any higher order terms in $d x, d y, d z$ or combinations thereof. The displacements $d x, d y$ and $d z$ should not be linked or confused with the mesh spacings $\Delta x, \Delta y, \Delta z$. They are free and should provide an interpolated expression for $a_{x}$ at any point inside a given mesh cell, e.g. a particle's position. We then use the difference approximations 


$$
\begin{gathered}
a_{x}{ }^{(2)}(x, y, z, \Delta x)=\frac{\partial \Phi}{\partial x}(x, y, z, \Delta x)=D_{x} \star \Phi, \\
\frac{\partial a_{x}}{\partial x}(x, y, z, \Delta x, \Delta y, \Delta z)=\frac{\partial^{2} \Phi}{\partial x^{2}}(x, y, z, \Delta x, \Delta y, \Delta z) \equiv D_{x x} \star \Phi, \\
\frac{\partial a_{x}}{\partial y}(x, y, z, \Delta x, \Delta y, \Delta z)=\frac{\partial^{2} \Phi}{\partial x \partial y}(x, y, z, \Delta x, \Delta y, \Delta z) \equiv D_{x y} \star \Phi, \\
\frac{\partial a_{x}}{\partial z}(x, y, z, \Delta x, \Delta y, \Delta z)=\frac{\partial^{2} \Phi}{\partial x \partial z}(x, y, z, \Delta x, \Delta y, \Delta z) \equiv D_{x z} \star \Phi .
\end{gathered}
$$

We may now generate a mesh sampled $a_{x}^{\dagger(2)}=\amalg a_{x}{ }^{(2)}$ in the standard way and integrate this over any volume containing one grid cell centre to obtain a set of acceleration values $a_{\mathrm{m}, \mathrm{x}}^{\dagger}{ }^{(2)}$ given by the following expressions:

$$
\begin{aligned}
a_{i j k, x}{ }^{(2)}(d x, d y, d z) & =\frac{\Phi_{i+1, j k}-\Phi_{i-1, j k}}{2 \Delta x} \\
& +\frac{\Phi_{i+1, j k}+\Phi_{i-1, j k}-2 \cdot \Phi_{i j k}}{(\Delta x)^{2}} \cdot d x \\
& +\frac{\Phi_{i+1, j+1, k}-\Phi_{i-1, j+1, k}+\Phi_{i-1, j-1, k}-\Phi_{i+1, j-1, k}}{4 \Delta x \Delta y} \cdot d y \\
& +\frac{\Phi_{i+1, j, k+1}-\Phi_{i-1, j, k+1}+\Phi_{i-1, j, k-1}-\Phi_{i+1, j, k-1}}{4 \Delta x \Delta z} \cdot d z
\end{aligned}
$$

Note that we generally take $\Delta x=\Delta y=\Delta z=1$ i.e. the cell-length is assumed to be equal along the three axes and unity. The accelerations in $y$-and $z$-direction are calculated analogously.

At first glance the interpolation scheme Eq. 15 for the acceleration seems to be closely related to the procedure used in a standard cloud-in-cell (CIC) scheme. In one dimension, CIC and SUPERBOX methods can be more easily compared to each other. For $0 \leq d x<\Delta x / 2$ one has

$$
\begin{aligned}
\text { CIC : } a(x+d x) & =a_{i} \cdot \frac{\Delta x-d x}{\Delta x}+a_{i+1} \cdot \frac{d x}{\Delta x} \\
& =\frac{\Phi_{i+1}-\Phi_{i-1}}{2 \Delta x}+\frac{\Phi_{i+2}-\Phi_{i+1}-\Phi_{i}+\Phi_{i-1}}{2(\Delta x)^{2}} d x, \\
\text { Superbox : } a(x+d x) & =a_{i}+\left.\frac{\mathrm{d} a}{\mathrm{~d} x}\right|_{i} \cdot d x \\
& =\frac{\Phi_{i+1}-\Phi_{i-1}}{2 \Delta x}+\frac{\Phi_{i+1}+\Phi_{i-1}-2 \Phi_{i}}{(\Delta x)^{2}} d x,
\end{aligned}
$$

where $i$ denotes the cell-index. Clearly this is different, because CIC uses accelerations in two neighbouring cells, while SUPERBOX takes acceleration and its derivative only in one cell. Therefore we propose to consider SUPERBOX as an NGP type scheme, although a second order interpolated acceleration is used, because nowhere 
the acceleration of the neighbouring cell is entering into the equations. Though of NGP nature, $a_{x}$ in SUPERBOX behaves steadily when crossing cell boundaries along the $x$-axis (and $a_{y}, a_{z}$ correspondingly on their respective axes). In contrast to CIC our scheme is not steady in any other direction. However, the errors induced by that are much smaller than in a standard NGP scheme due to the above higher order interpolation, and are perfectly tolerable as will be shown throughout this paper.

The salient feature of SUPERBOX regarding the force anisotropies can be understood by considering the force at a distance $r=|\mathbf{d r}|$ from a particle located at the centre of a cell. To lowest order the SUPERBOX force-error $\varepsilon$ is then

$$
\vec{\varepsilon}=-\frac{\mathbf{d r}}{r^{3}},
$$

where $\mathbf{d r}=(d x, d y, d z)$ is the displacement vector from the cell centre, and we have neglected all terms of second order in $\Delta x, \Delta y$, or $\Delta z$ in a Taylor series expansion of the potential differences. In other words to lowest order the SUPERBOX force error points to the exact centre of the cell. Thus, compared with standard NGP schemes, we get a very precise force-calculation in SUPERBOX, as shown in Figs. 2 and 3.
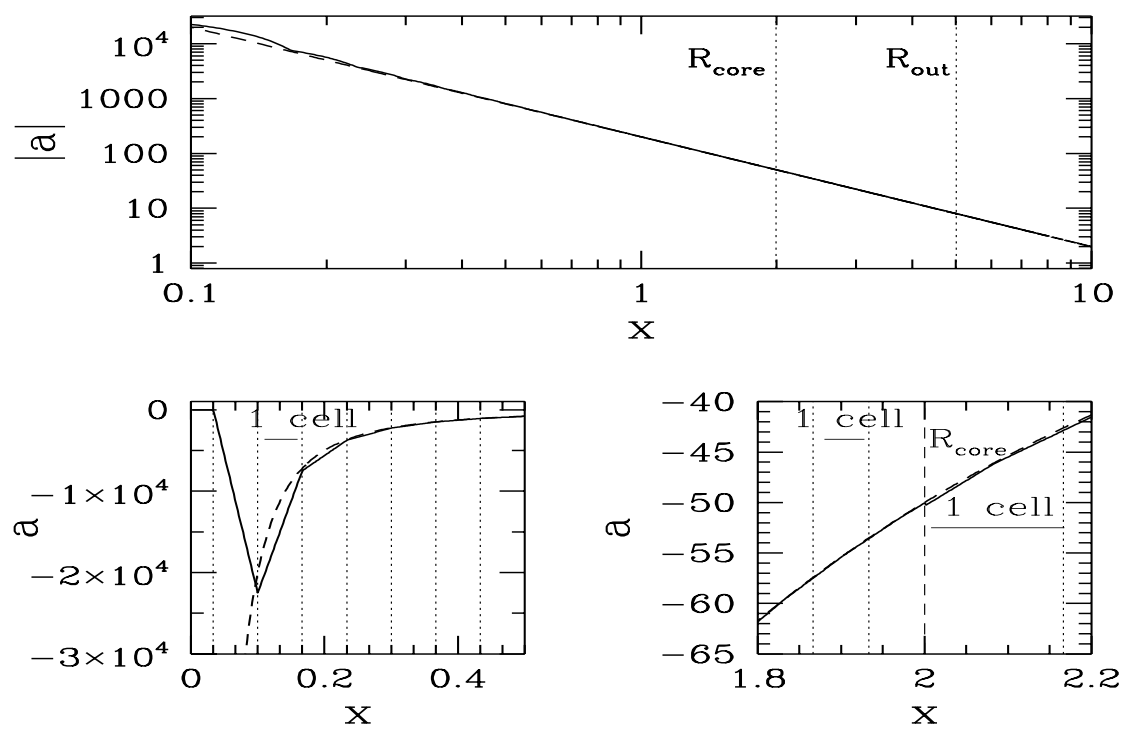

Fig. 2. Pairwise acceleration calculated by SUPERBox (using $H_{000}=1$ ) for one particle fixed in the centre and the second moving along the $x$-axis. The agreement between the acceleration calculated by SUPERBOX (solid line) and the analytical (i.e. Keplerian) force (dashed line) is very good, if the two particles are at least 2 cells apart. Upper panel: pairwise acceleration over the entire spatial range. Lower panel: blown up pictures for the innermost part (left) and the grid-boundary (right). 

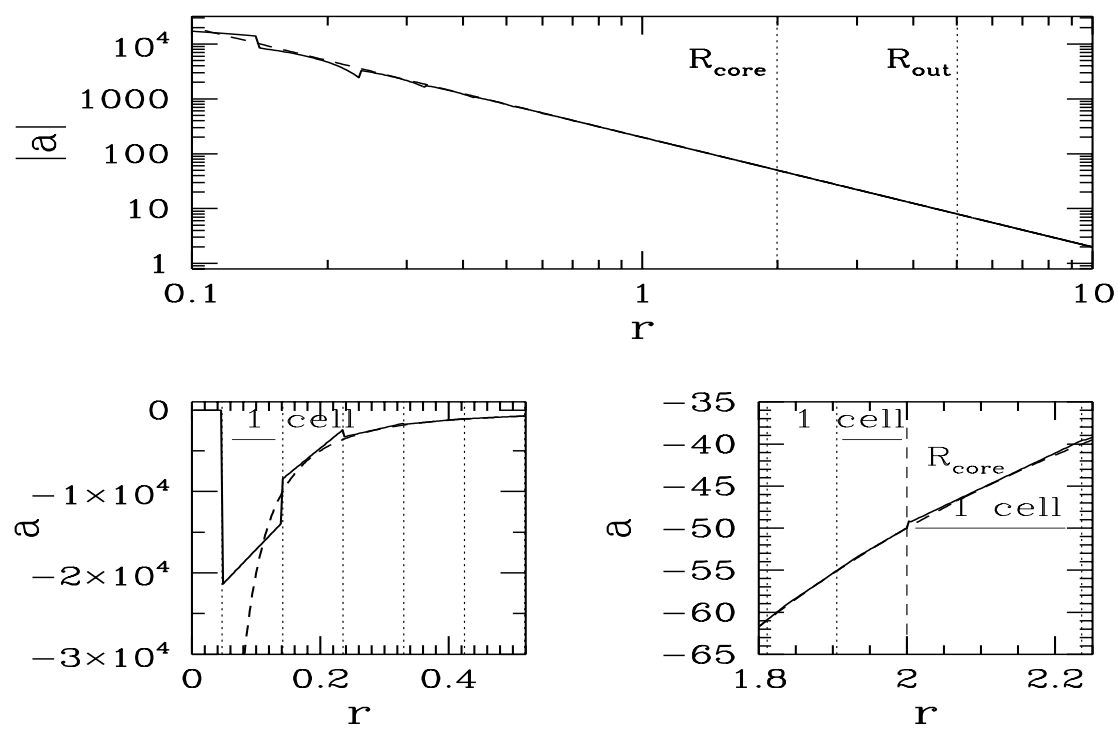

Fig. 3. As Fig. 2, except that here the second particle moves along a diagonal with $y=x, z=0, r=\sqrt{x^{2}+y^{2}}$.

The orbits of the particles are integrated forward in time using the leapfrog scheme. For example, for the $x$-components of the velocity, $v_{x}$, and position, $x$, vectors of particle $l$,

$$
\begin{aligned}
v_{x, l}^{n+1 / 2} & =v_{x, l}^{n-1 / 2}+a_{x, l}^{n} \cdot \Delta t \\
x_{l}^{n+1} & =x_{l}^{n}+v_{x, l}^{n+1 / 2} \cdot \Delta t,
\end{aligned}
$$

where $n$ denotes the $n$th time-step and $\Delta t$ is the length of the integration step.

\subsection{The grids}

For each galaxy, 5 grids with 3 different resolutions are used. This is made possible by invoking the additivity of the potential (Fig. 4).

The five grids are as follows:

- Grid 1 is the high-resolution grid which resolves the centre of the galaxy. It has a length of $2 \times R_{\text {core }}$ in one dimension. In evaluating the densities, all particles of the galaxy within $r \leq R_{\text {core }}$ are stored in this grid.

- Grid 2 has an intermediate resolution to resolve the galaxy as a whole. The length is $2 \times R_{\text {out }}$, but only particles with $r \leq R_{\text {core }}$ are stored here, i.e. the same particles as are also stored in grid 1 . 


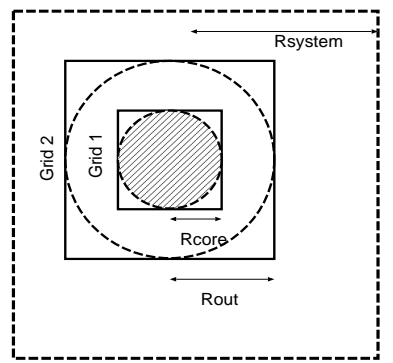

Grid $1+2$

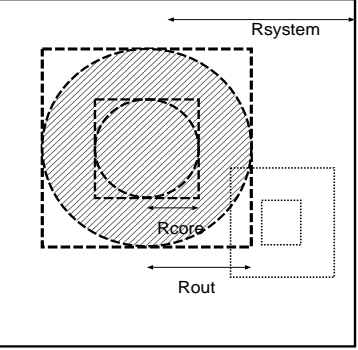

Grid 4

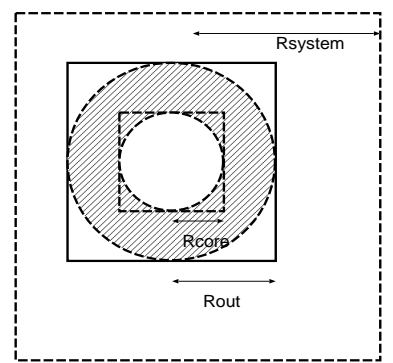

Grid 3

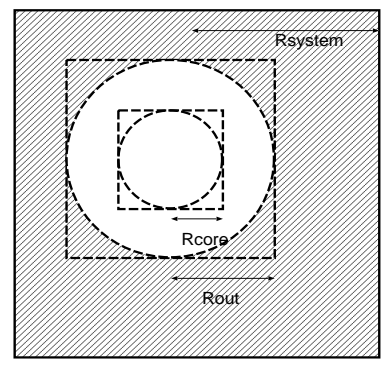

Grid 5

Fig. 4. The five grids of SUPERBox. In each panel, solid lines highlight the relevant grid. Particles are counted in the shaded areas of the grids. The lengths of the arrows are $(N / 2)-2$ grid-cells (see text). In the bottom left panel, the grids of a hypothetical second galaxy are also shown as dotted lines.

- Grid 3 has the same size and resolution as grid 2, but it only contains particles with $R_{\text {core }}<r \leq R_{\text {out }}$.

- Grid 4 has the size of the whole simulation area (i.e. 'local universe' with $2 \times$ $R_{\text {system }}$ ), and has the lowest resolution. It is fixed. Only particles of the galaxy with $r \leq R_{\text {out }}$ are stored in grid 4 .

- Grid 5 has the same size and resolution as grid 4. This grid treats the escaping particles of a galaxy, and contains all particles with $r>R_{\text {out }}$.

Grids 1 to 3 are focused on a common centre of the galaxy and move with it through the 'local universe', as detailed below. All grids have the same number of cells per dimension, $N$, for all galaxies. The boundary condition, requiring two empty cells with $\varrho=0$ at each boundary, is open and non-periodic, thus providing an isolated system. This however means that only $N-4$ active cells per dimension are used. To keep the storage requirement low, all galaxies are treated consecutively in the same grid-arrays, whereby the particles belonging to different galaxies can have different masses. Each of the 5 grids has its associated potential $\Phi_{\mathrm{i}}, i=1,2, \ldots, 5$ computed by the PM technique from the particles of one galaxy located as described above. The accelerations are obtained additively from the 5 potentials of each galaxy in turn in the following way:

$$
\begin{aligned}
\Phi(r) & =\left[\theta\left(R_{\text {core }}-r\right) \cdot \Phi_{1}+\theta\left(r-R_{\text {core }}\right) \cdot \Phi_{2}+\Phi_{3}\right] \cdot \theta\left(R_{\text {out }}-r\right) \\
& +\theta\left(r-R_{\text {out }}\right) \cdot \Phi_{4}+\Phi_{5} \\
\Phi\left(R_{\text {core }}\right) & =\Phi_{1}+\Phi_{3}+\Phi_{5} \\
\Phi\left(R_{\text {out }}\right) & =\Phi_{2}+\Phi_{3}+\Phi_{5}
\end{aligned}
$$


where $\theta(\xi)=1$ for $\xi>0$ and $\theta(\xi)=0$ otherwise. This means:

- For a particle in the range $r \leq R_{\text {core, }}$, the potentials of grids 1,3 and 5 are used to calculate the acceleration.

- For a particle with $R_{\text {core }}<r \leq R_{\text {out }}$, the potentials of grids 2,3 and 5 are combined.

- And finally, if $r>R_{\text {out }}$ then the acceleration is calculated from the potentials of grids 4 and 5 .

- A particle with $r>R_{\text {system }}$ is removed from the computation.

Due to the additivity of the potential (and hence its derivatives, the accelerations) the velocity changes originating from the potentials of each of the galaxies can be separately updated and accumulated in the first of the leap-frog formulas Eq. 17. The final result does not depend on the order by which the galaxies are taken into account and it could be computed even in parallel, if a final accumulation takes place. After all velocity changes have been applied in all galaxies, the positions of the particles are finally updated (see Fig. 1).

As long as the galaxies are well separated, they feel only the low-resolution potentials of the outer grids. But as the galaxies approach each other their high-resolution grids overlap, leading to a high-resolution force calculation during the interaction.

\subsubsection{Grid tracking}

Two alternative schemes to position and track the inner and middle grids can be used. The most useful scheme centres the grids on the density maximum of each galaxy at each step. The position of the density maximum is found by constructing a sphere of neighbours centred on the densest region, in which the centre of mass is computed. This is performed iteratively. The other option is to centre the grids during run-time on the position of the centre of mass of each galaxy using all its particles remaining in the computation.

\subsubsection{Edge-effects}

It is shown in Fig. 4 that only spherical regions of the cubic grids contain particles (except for Grid 5). Particles with eccentric orbits can cross the border of two grids, thus being subject to forces resolved differently. No interpolation of the forces is done at the grid-boundaries. This keeps the code fast and slim, but the grid-sizes have to be chosen properly in advance to minimise the boundary discontinuities. As shown in Section 4, this leads to some additional but negligible relaxationeffects, because the derived total potential has insignificant discontinuities at the grid-boundaries (Wassmer 1992). The best way to avoid these edge-effects is to place the grid-boundaries at 'places' where the slope of the potential is not steep.

Fig. 5 shows that, as long as the grid-sizes are chosen properly, there are no serious effects at the grid-boundaries. Only in the example shown in the bottom-right panel 
the innermost grid was chosen too small, leading to a low particle number per gridcell. Performing such computations with $H_{000}=4 / 3$ leads to spurious results, as explained in Section 2.1. Nevertheless the profile is smooth at the grid-boundary.
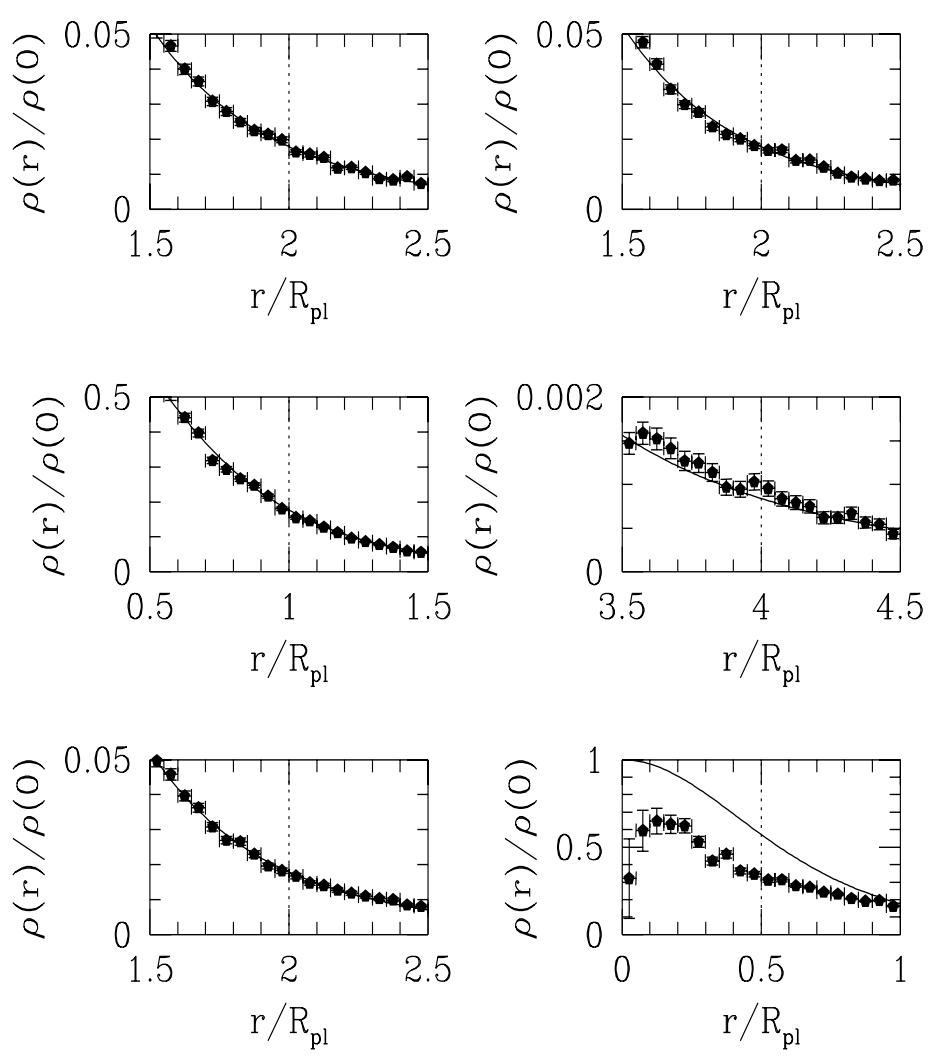

Fig. 5. The radial density distribution of Plummer spheres at the grid-boundary between the innermost and the middle grid (shown as dotted vertical lines) after computing for 10 half-mass crossing times for different grid-sizes. The radial density profile resulting from the SUPERBoX computation, always with $N_{\mathrm{p}}=5 \times 10^{4}$, is shown as dots with error-bars. The analytical density law is shown as the solid line. In all cases except the bottom left panel $R_{\text {out }}=9 R_{\mathrm{pl}}$ (where $R_{\mathrm{pl}}$ is the Plummer radius). Upper left: $N=32$ grids per dimension, $R_{\text {core }}=2 R_{\mathrm{pl}}$. Upper right: $N=64, R_{\text {core }}=2 R_{\mathrm{pl}}$. Middle left: $N=32, R_{\text {core }}=1 R_{\mathrm{pl}}$. Middle right: $N=32, R_{\text {core }}=4 R_{\mathrm{pl}}$. Bottom left: $N=32$, $R_{\text {core }}=2 R_{\mathrm{pl}}$ and $R_{\text {out }}=14 R_{\mathrm{pl}}$. Bottom right: $N=32, R_{\text {core }}=0.5 R_{\mathrm{pl}}$. Only if the innermost grid is chosen too small (bottom right) are there significant deviations from the analytical profile. For this calculations $H_{000}=4 / 3$ is used.

\subsubsection{Model units}

SUPERBOX employs model units, with the gravitational constant in model units being $G_{\text {mod }}=2$ owing to historical reasons. A flag specifies if the user wants to input and output data in physical units (i.e. $\mathrm{kpc}, \mathrm{M}_{\odot}, \mathrm{km} / \mathrm{s}, \mathrm{Myr}$ ). Scaling to and 
from physical units is achieved via the formulae:

$$
\begin{aligned}
\left(\frac{L_{\text {phys }}}{L_{\text {mod }}}\right)^{3} & =\frac{G_{\text {phys }}}{G_{\text {mod }}} \frac{M_{\text {phys }}}{M_{\text {mod }}}\left(\frac{T_{\text {phys }}}{T_{\text {mod }}}\right)^{2}, \\
\frac{V_{\text {phys }}}{V_{\text {mod }}} & =\frac{L_{\text {phys }}}{T_{\text {phys }}} \frac{T_{\text {mod }}}{L_{\text {mod }}},
\end{aligned}
$$

where $L$ is the length, $M$ the mass, $T$ the time, $V$ the velocity and $G$ the gravitational constant. The model length unit is taken to be $L_{\bmod }=1$ for the length of $R_{\text {core }, 1}$ of galaxy 1 . The corresponding physical length scale, $L_{\text {phys }}$, is taken from the input data that specifies the length of this grid. The physical mass of the first galaxy, $M_{\text {phys }, 1}$, is taken to be unity in model units, $M_{\text {mod,1 }}=1$. The integration

step-size, $T_{\text {phys }}$, is specified by the user. The step-size in model units, $T_{\text {mod }}$, follows from Eq. 19.

Converting factors for each grid of all galaxies are calculated to get the forces and accelerations, due to the FFT and the differentiation of the potentials requiring a cell-length of unity.

\section{Conservation of Energy and Angular Momentum}

In order to check how well energy and angular momentum are conserved, calculations with isolated Plummer-spheres in virial equilibrium with different particle numbers, time-steps and grid-resolutions are presented here. To have a nonvanishing angular momentum vector in $z$-direction for the isolated galaxy model, all particle orbits are taken to have the same direction in the $x y$-plane. This procedure does not change any other properties of the Plummer-model, but makes it possible to check for relative changes in angular momentum.

\subsection{Total Energy}

Calculating the correct potential energy of a stellar system requires summing all two-body interactions between all particles. This is quite impossible for $N_{\mathrm{p}} \gtrsim 10^{6}$. By using the available grid-based approximation and adding up only the mean potential values of the cells for each particle, a useful estimate of the total potential energy is obtained. If, on the other hand, the deviation from the centre of the cell and contributions from the neighbouring cells were taken into account to obtain a more accurate estimate, then CPU-time would have to be used for an arguably purposeless endeavour. For simplicity, in SUPERBOX the total potential energy is computed without any interpolation inside grid cells (as it was used for the acceleration); only the potential value of the particle's cells are taken into account to compute the potential energy. The total potential energy at the $n$th time-step is thus 


$$
E_{\mathrm{pot}}^{n} \approx-\frac{1}{2} \sum_{i=1}^{N_{\mathrm{p}}} m_{i} \Phi^{n}\left(x_{i}, y_{i}, z_{i}\right),
$$

where $\Phi^{n}$ is the sum of the potentials of all grids and galaxies used to calculate the force on particle $i$ at the $n$-th time-step, or in other words, all potentials which contribute to the leap-frog update of the velocity in Eq. 17. $m_{i}$ is the mass of the particle (equal for all particles belonging to the same galaxy).

The total kinetic energy is also calculated only approximately during runtime. This comes about because in the leapfrog integration-scheme the velocities are half a time-step behind the positions. Within our integration scheme the time-centred interpolation for the kinetic energy would be

$$
E_{\text {kin }}^{n}=\frac{1}{2} \sum_{i=1}^{N_{\mathrm{p}}} m_{i} \sum_{j=1}^{3}\left(\frac{v_{i, j}^{n+1 / 2}+v_{i, j}^{n-1 / 2}}{2}\right)^{2},
$$

where $v_{i, j}^{n}$ is the $j$-component of the velocity vector of particle $i$ at time-step $n$. In SUPERBOX the philosophy is to keep the amount of storage as small as possible, so that only the current velocities are stored. To keep the error in $E_{\text {kin }}$ as small as possible, particle kinetic energies are calculated before and after the velocity vectors are updated in one time-step. From these two values the arithmetic average is taken:

$$
E_{\mathrm{kin}}^{n} \approx \frac{1}{4} \sum_{i=1}^{N_{\mathrm{p}}} m_{i} \sum_{j=1}^{3}\left(\left(v_{i, j}^{n-1 / 2}\right)^{2}+\left(v_{i, j}^{n+1 / 2}\right)^{2}\right) .
$$

This still implies an additional error of the order of $(\Delta v)^{2}$, but keeps storage low and the code fast.

To quantify the performance of SUPERBOX in terms of conservation of total energy, $E_{\text {tot }}(t)=E_{\text {pot }}(t)+E_{\text {kin }}(t)$, calculations with different time-steps, particle number and grid resolution are done. We consider the relative change $\Delta E_{\text {tot }}(t) / E_{\text {tot }}\left(t_{0}\right)$, where $\Delta E_{\text {tot }}(t)=E_{\text {tot }}(t)-E_{\text {tot }}\left(t_{0}\right)$, and $t_{0}$ is the reference time. It is chosen to be zero when the adjustment to equilibrium after setting up the discrete rendition of the galaxy is accomplished. $\operatorname{Max}\left(\Delta E_{\text {tot }}(t) / E_{\text {tot }}(0)\right)$ denotes the largest deviation in energy that occurs during a computation (see Fig. 6).

Table 1 shows that deviations in $E_{\text {tot }}(t)$ are less than 0.5 per cent, as long as the time-step, $\Delta t \lesssim T_{\text {cr }} / 10$, where $T_{\text {cr }}$ is the half-mass crossing-time of the Plummermodel. We consider $\Delta t \leq T_{\text {cr }} / 50$ to be a safe choice. In theory (see Hockney \& Eastwood 1981) the error of the leapfrog integrator should decrease as $(\Delta t)^{2}$ for $\Delta t \rightarrow 0$, but as is evident from Table 1 , other error sources become prominent at a sufficiently small time step. 
Table 1

Maximum deviation in total energy, $\max \left(\Delta E_{\mathrm{tot}} / E_{\mathrm{tot}}\right)$ after $10 T_{\mathrm{cr}}$ for different time-steps using $N_{\mathrm{p}}=10^{5}$ particles and $N=32$ grids per dimension.

\begin{tabular}{l|c}
$\Delta t$ (time-step) & $\max \left(\Delta E_{\mathrm{tot}}(t) / E_{\mathrm{tot}}(0)\right)$ \\
\hline$T_{c r} / 10$ & $0.4 \%$ \\
$T_{c r} / 100$ & $0.2 \%$ \\
$T_{c r} / 1000$ & $0.2 \%$
\end{tabular}

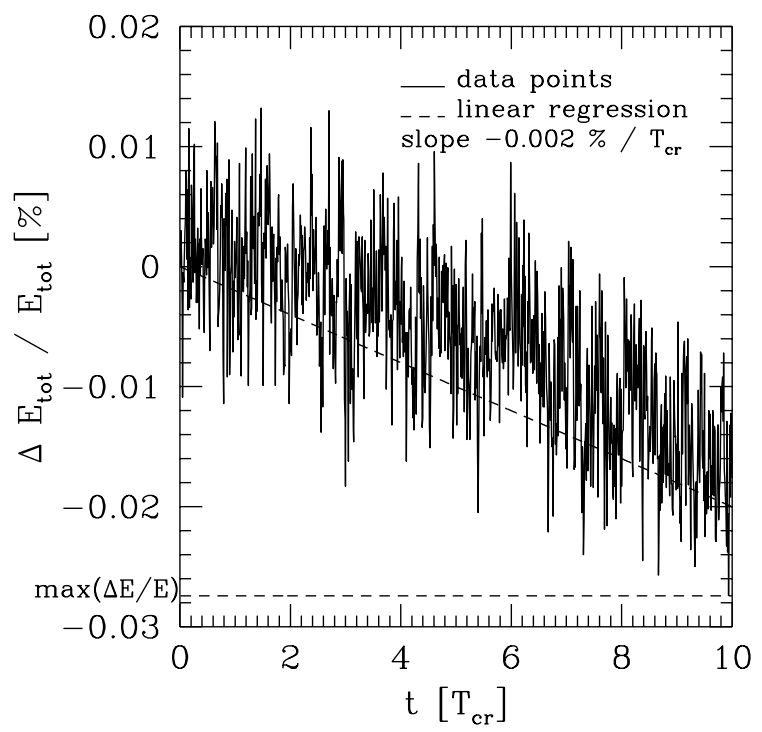

Fig. 6. Linear drift of the total energy $\Delta E_{\text {tot }}(t) / E_{\text {tot }}(0)$ in per cent for $N_{\mathrm{p}}=10^{6}$ particles and $N=64$ grids per dimension using $\Delta t=T_{\mathrm{cr}} / 100$ (the regression line is shown with a little offset for clarity).

Fig. 6 shows the time evolution of the change in total energy for a particular calculation. A linear drift fits the data well, which holds true in computations extending over 50 crossing-times. The slope of the energy-drift is 0.002 per cent per crossingtime. The dispersion in energy is artificial due to the crude calculation of potential energy. Over a Hubble time, which corresponds to $150 \times T_{\mathrm{cr}}$ if the Plummer model galaxy is assumed to have $T_{\mathrm{cr}}=10^{8} \mathrm{yr}$, the resulting energy error is 0.3 per cent. This linear drift is intrinsic to a PM code (see Hockney \& Eastwood, their fig. 9.4), and its strength depends mainly on the choice of $H_{000}$ in the Green's function. $H_{000}$ determines the strength of the gravitational interaction of particles in the same cell, and also the self-gravitation of a particle (see Section 2.1). Additional errors arise through the limited grid-resolution and the approximations adopted in evaluating the energies. This gives an additional oscillation around the value of the linear drift seen in Fig. 6.

Increasing the number of particles per galaxy to $N_{\mathrm{p}}>10^{5}$ leads to little further improvement in $\max \left(\Delta E_{\text {tot }} / E_{\text {tot }}\right)$ with $N=32$. The decrease of the error levels 


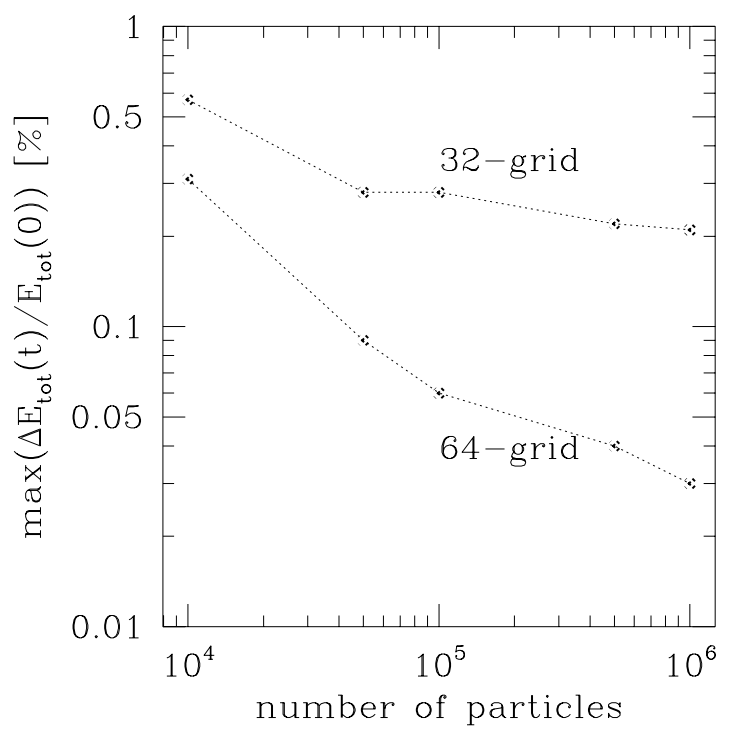

Fig. 7. Maximum deviation in energy, $\max \left(\Delta E_{\mathrm{tot}}(t) / E_{\mathrm{tot}}(0)\right)$ in per cent, after $10 T_{\mathrm{cr}}$, for different particle numbers and grid-resolutions, using $\Delta t=T_{\mathrm{cr}} / 100$.

off if the mean number of particles per cell becomes sufficiently large. However, changing the grid resolution from $N=32$ to 64 cells per dimension gives an improvement by a factor of at least 4 , with the decrease in error still progressing for $N_{\mathrm{p}}>10^{6}$ (Fig. 7). This is due to the estimate of the true potential by our grid-based potential, and thus of the potential energy, improving with increasing $N$. It also shows that increasing the number of grid-cells while keeping the number of particles low (with $H_{000}=4 / 3$ ) does not improve energy conservation (e.g. $\left.N=64, N_{\mathrm{p}}=10^{4}\right)$.

\subsection{Angular Momentum}

To calculate the absolute value of the total angular momentum of the galaxy, $L_{\mathrm{tot}}$, the same technique as for the kinetic energy is applied. That is, the arithmetic mean of the two velocity values (before and after the velocity update) is calculated for each velocity component to obtain an estimate of the velocity vector that is in-phase with the position vector.

The time evolution of $\Delta L_{\mathrm{tot}}(t) / L_{\mathrm{tot}}(t)$ for one particular calculation is shown in Fig. 8. As with $E_{\text {tot }}$, computations over $50 T_{\text {cr }}$ show insignificant deviations from the linear drift. The slope of the drift is about 0.003 per cent per crossing-time.

Conservation of $L_{\text {tot }}$ does not depend on $\Delta t$ as long as the time-step is small enough ( $\Delta t \leq T_{\text {cr }} / 50$ as for energy in Section 3.1). But it is highly dependent on the gridresolution. Changing from $N=32$ to 64 grids per dimension improves the change 


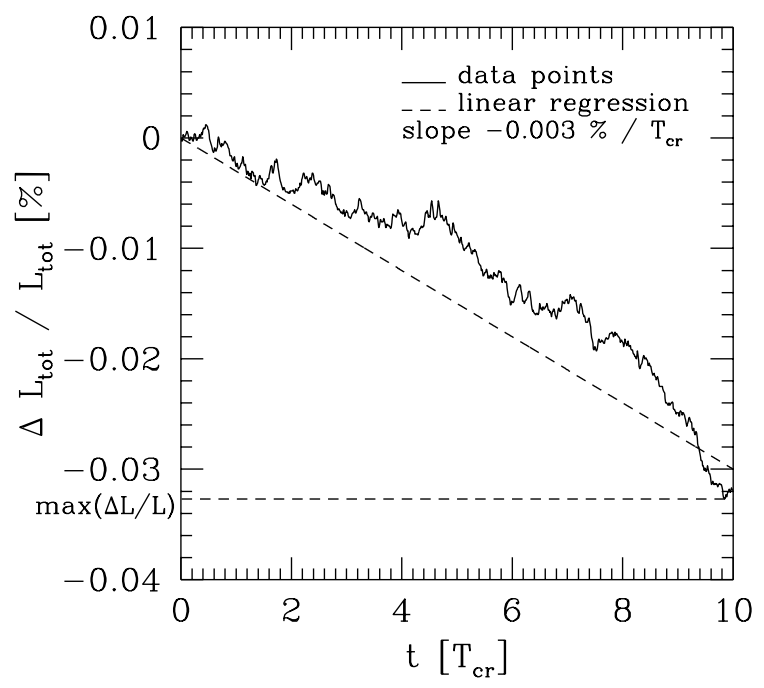

Fig. 8. The change in $\Delta L_{\text {tot }}(t) / L_{\text {tot }}(0)$ in per cent for $N_{\mathrm{p}}=10^{6}$ particles and $N=64$ grids per dimension using $\Delta t=T_{\mathrm{cr}} / 100$ (the regression is shown offset).

in angular momentum by at least a factor of 10. From Fig. 9 it can be seen that $\max \left(\Delta L_{\text {tot }} / L_{\text {tot }}\right)$ is quite independent of $N_{\mathrm{p}}$.

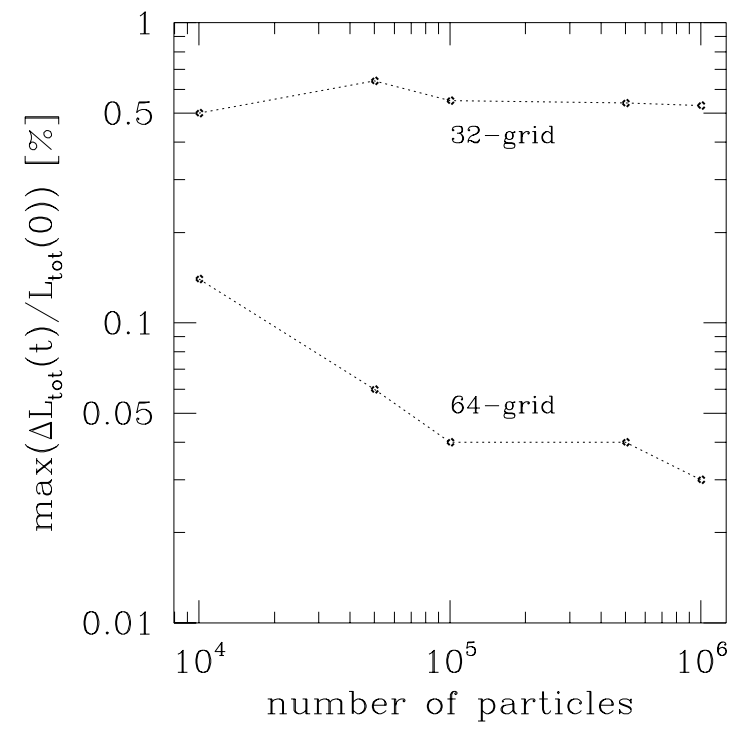

Fig. 9. Maximum deviation in angular momentum, $\max \left(\Delta L_{\text {tot }}(t) / L_{\text {tot }}(0)\right)$ in per cent, after $10 T_{\mathrm{cr}}$, for different particle numbers and grid-resolutions, using $\Delta t=T_{\mathrm{cr}} / 100$. $\max \left(\Delta L_{\text {tot }}(t) / L_{\text {tot }}(0)\right)$ is defined analogously to $\max \left(\Delta E_{\text {tot }}(t) / E_{\text {tot }}(0)\right)$ in Section 3.1. 


\section{Relaxation}

Galaxies have two-body relaxation times of the order of $10^{7} \mathrm{Gyr}$, and show little or no relaxation over a Hubble time even in their inner parts. Therefore, a programme which models galaxies has to be collision-less. Since no particle-mesh code can be entirely free of relaxation, we have to check on which time-scales SUPERBOX provides reliable results.

Following Standish \& Aksnes (1969), the following experiment is performed: A number of equal-mass and fixed particles is distributed homogeneously inside a sphere of radius $R_{\mathrm{sph}}$ with a constant density distribution. A second group of particles is distributed on the surface of this sphere. They are allowed to move through the centre and leave the sphere on the opposite side. To make sure they leave the sphere, they are given an initial non-zero radial velocity component towards the centre of the sphere. The points where the moving particles leave the sphere are noted and the calculation is stopped after all moving particles have left the sphere. For every particle, its deflection angle $\alpha$ is obtained from

$$
\cos \alpha=-\frac{\vec{R}_{I} \vec{R}_{F}}{\left|\vec{R}_{I}\right|\left|\vec{R}_{F}\right|}
$$

where $\vec{R}_{I}$ and $\vec{R}_{F}$ are the vectors from the centre of the sphere to the initial and

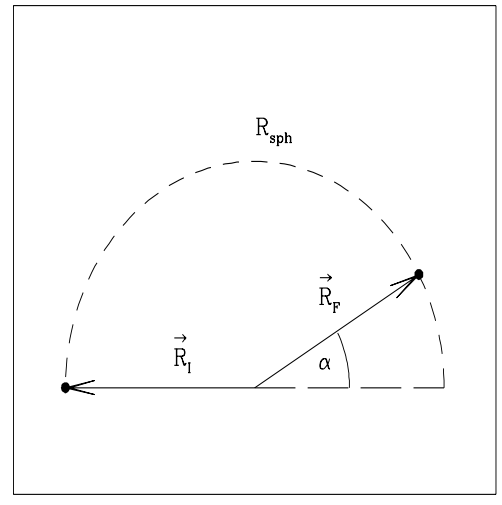

Fig. 10. The deflection angle $\alpha$.

final position of the particle (see Fig. 10). The mean deflection angle, $\bar{\alpha}$, of all particles is computed and the relaxation time, $T_{\text {rel }}$, is calculated from

$$
T_{\mathrm{rel}}=\frac{\sin 90}{\sin \bar{\alpha}} \bar{T}_{\mathrm{cr}}
$$

Here $\bar{T}_{\mathrm{cr}}$ is the mean time the moving particles require to travel through the sphere. The number of moving particles is chosen to be $10^{5}$, which gives a sufficiently 
accurate estimate for $T_{\text {rel }}$. The number of fixed particles, $N_{\text {fix }}$, and the size of the inner mesh, $R_{\text {core }}$, are varied. Grids with 32 grid-cells per dimension are used. For a certain combination of $N_{\text {fix }}$ and $R_{\text {core }}, 100$ calculations with different random number seeds (both for the fixed and moving particles) are performed. In all cases, $R_{\text {sph }}<R_{\text {out }}$ (see also Fig. 4).

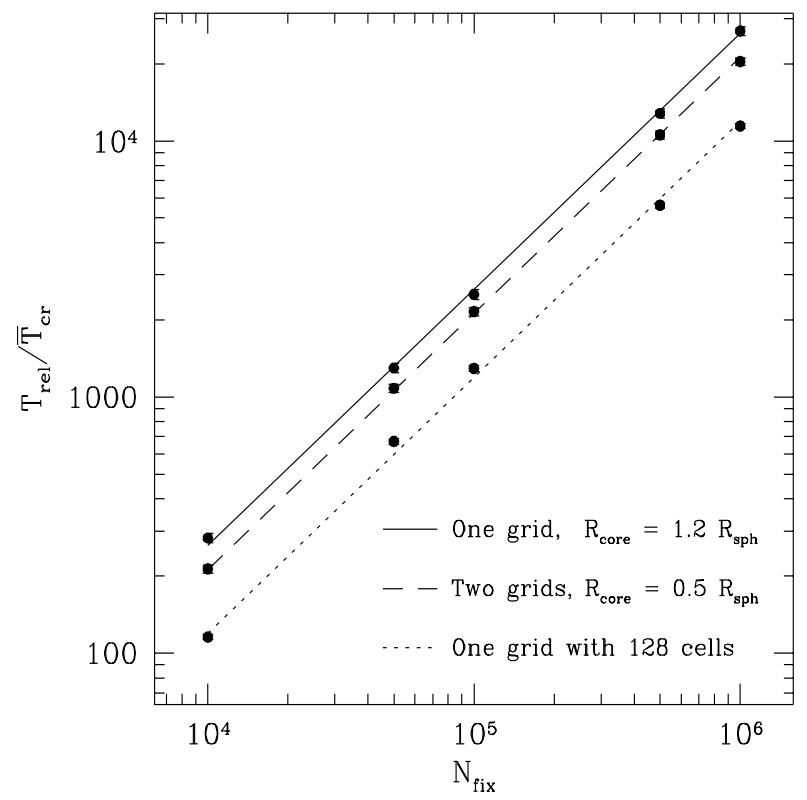

Fig. 11. The ratio $T_{\text {rel }} / \bar{T}_{\text {cr }}$ as a function of the number of fixed particles.

Fig. 11 shows the ratio $T_{\text {rel }} / \bar{T}_{\text {cr }}$ as a function of $N_{\text {fix }}$. If one grid contains the whole sphere $\left(R_{\text {core }}=1.2 R_{\text {sph }}\right)$ a linear dependence of $T_{\text {rel }} / \bar{T}_{\text {cr }}$ on $N_{\text {fix }}$ fits the results very well. Such a dependency is predicted for large particle numbers by standard relaxation theory (Spitzer \& Hart 1971; Binney \& Tremaine 1987). For $N_{\text {fix }} \gtrsim 10^{5}$, $T_{\text {rel }} / \bar{T}_{\mathrm{cr}}>10^{3}$. Since $T_{\mathrm{cr}} \approx 10^{8} \mathrm{yr}$ for a typical galaxy, the relaxation time is one order of magnitude larger than a Hubble time. Hence galaxies can be modelled with SUPERBOX provided $N_{\mathrm{p}}>10^{5}$ particles per galaxy.

These results are not substantially changed if the particles have to cross a grid boundary. As an example, the case $R_{\text {core }}=0.5 R_{\text {sph }}$ is shown in Fig. 11. Compared to the one-grid case, $T_{\text {rel }}$ is reduced by about 20 per cent nearly independently of $N_{\text {fix }}$. Hence, grid boundaries do not decrease $T_{\text {rel }}$ significantly. The relaxation time also drops if the number of grid-cells is increased: Due to the better resolution, forces from particles in adjacent cells become larger, increasing the relaxation. As an example the case with 128 cells per dimension is shown in Fig. 11. The relaxation times are a factor of 2.3 smaller compared to the case with $N=32$. Assuming that all particles within a cell are located at the cell centre, and treating the deflection of the moving particles as a pure N-body problem, we may obtain an analytical estimate of the relaxation in SUPERBOX. A consideration similar to the one described in Binney \& Tremaine (1987) p. $187 \mathrm{ff}$ shows that the relaxation time should depend in the following way on $N_{\text {fix }}$ and $N_{\mathrm{gc}}$ : 


$$
T_{\mathrm{rel}}=\kappa \cdot \frac{N_{\mathrm{fix}}}{\ln \left(\gamma N_{\mathrm{gc}}\right)} \cdot T_{\mathrm{cr}}
$$

with $\gamma=1 / 3$. From the experiments described in this section we obtain a value for the constant of proportionality $\kappa=0.05$. Together with this value, Eq. 25 gives an estimate of the relaxation time of SUPERBOX. From this result we can quantify, for a given grid resolution and computational time, the minimum number of particles that should be used to exclude any unwanted relaxation effects. It is again obvious that calculations with a high number of grid-cells should be done only with a sufficiently large particle number to ensure $T_{\text {rel }}>T_{\text {Hubble }}$.

\section{Memory and CPU-Time Requirements}

\subsection{Memory}

The memory requirement of SUPERBOX scales both linearly with the particle number, $N_{\mathrm{p}}$, and with the number of grid-cells, $N_{\mathrm{gc}}=N^{3}$. For the particle array, 24 byte per particle ( 4 bytes per phase-space variable) are needed. The total amount of memory for different particle numbers and grid-resolutions is shown in Fig. 12.
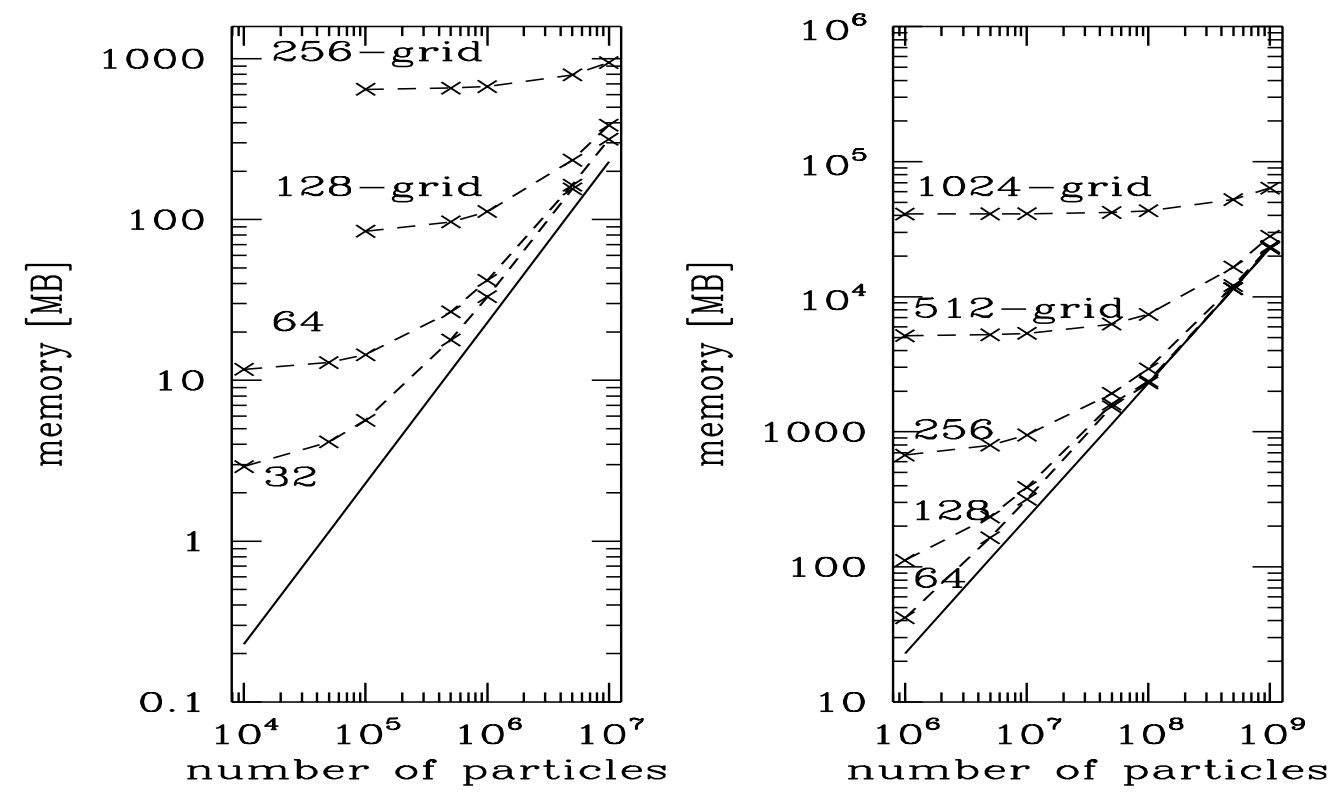

Fig. 12. The total memory requirement of SUPERBOX for different grid-resolutions as function of the particle-number $N_{\mathrm{p}}$ (straight line is the memory requirement of the particle array alone). 
The memory required for a SUPERBOX calculation can be estimated from

$$
\begin{aligned}
\text { memory grid }_{\text {ge }} & =\left(5 \cdot N^{3}+N(2 N)^{2}+(N+1)^{3}\right) \times 4 \text { byte, } \\
\text { memory }_{\text {particles }} & =\left(8 \cdot N_{\mathrm{p}}+\text { overhead }\right) \times 4 \text { byte }
\end{aligned}
$$

The first term on the right-hand side of Eq. 26 comes from the 5 grids (Section 2.3). The second term comes from the array needed for the FFT, and the last term comes from the array required to store the Fourier-transformed Green's function. The memory usage of the grids is independent of the number of galaxies, since they are treated consecutively in the same grid-arrays. The 'overhead' contains the arrays of the SUPERBOX parameters (e.g. grid sizes), the centre of mass and density, and output data such as energies, angular momenta and Lagrangian radii, for each galaxy. Increasing the number of galaxies, while keeping the total particle and grid number constant, reduces the relative overhead contribution (Eq. 27) slightly due to these large arrays scaling only with the number of particles per galaxy.

\subsection{CPU-time}

The time-step cycle of SUPERBOX is divided into three main routines (Fig. 1). Firstly, in GETRHO the mass density of the grids is calculated. Secondly, the FFT routine computes the potential on the grids. Thirdly, the PUSHER routine contains the force calculation, the position and velocity updating, and collection of the output data. These three routines need about 99 per cent of the total CPU time. Fig. 13 shows the amount of CPU-time per step, $t_{\mathrm{CPU}}$, needed for the different routines. All data are derived on a Pentium II $400 \mathrm{MHz}$ processor with Linux as the operating system and the GNU-g77 Fortran Compiler (with options $-\mathrm{O} 3-\mathrm{m} 486$ ) for a model single galaxy. For the GETRHO and PUSHER routines, $t_{\mathrm{CPU}} \propto N_{\mathrm{p}}$, while for the FFT routine, $t_{\mathrm{CPU}} \propto N_{\mathrm{gc}} \log _{10} N_{\mathrm{gc}}\left(N_{\mathrm{gc}}=N^{3}\right.$; Fig. 14), which is by far the dominant contribution. The resultant CPU time per step for galaxy $i$ is:

$$
t_{\mathrm{CPU}, i}=\alpha N_{p, i}+\beta \times 5 N_{\mathrm{gc}} \log _{10} N_{\mathrm{gc}} .
$$

As another example, for a Pentium $200 \mathrm{MHz}$ MMX, $t_{\mathrm{CPU}} \approx 20 \mathrm{sec} / \mathrm{step}$ for $N_{\mathrm{p}}=$ $10^{6}$ particles in one galaxy and $N_{\mathrm{gc}}=64^{3}$ grids.

The CPU-time needed for a computation with $N_{\text {gal }}$ galaxies is derived by adding the individual times,

$$
t_{\mathrm{CPU}, \text { tot }}=\sum_{i=1}^{N_{\mathrm{gal}}} t_{\mathrm{CPU}, i} .
$$



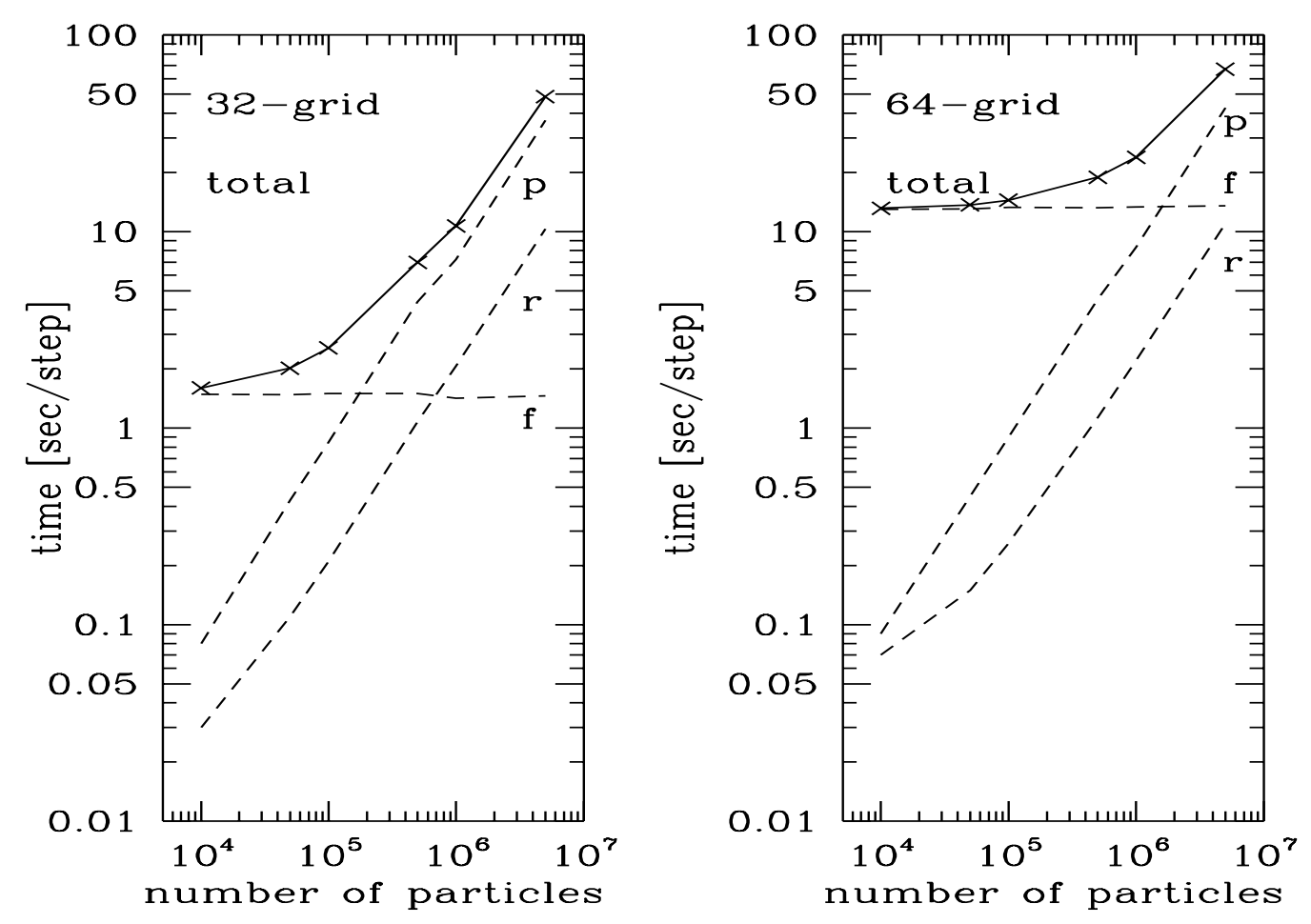

Fig. 13. Contributions to the CPU time, $t_{\mathrm{CPU}}$, by different routines, and total CPU-time per step for different particle numbers and grid resolutions ( $r$ denotes the GETRHO-routine, $f$ the FFT-routine and $p$ the PUSHER-routine).

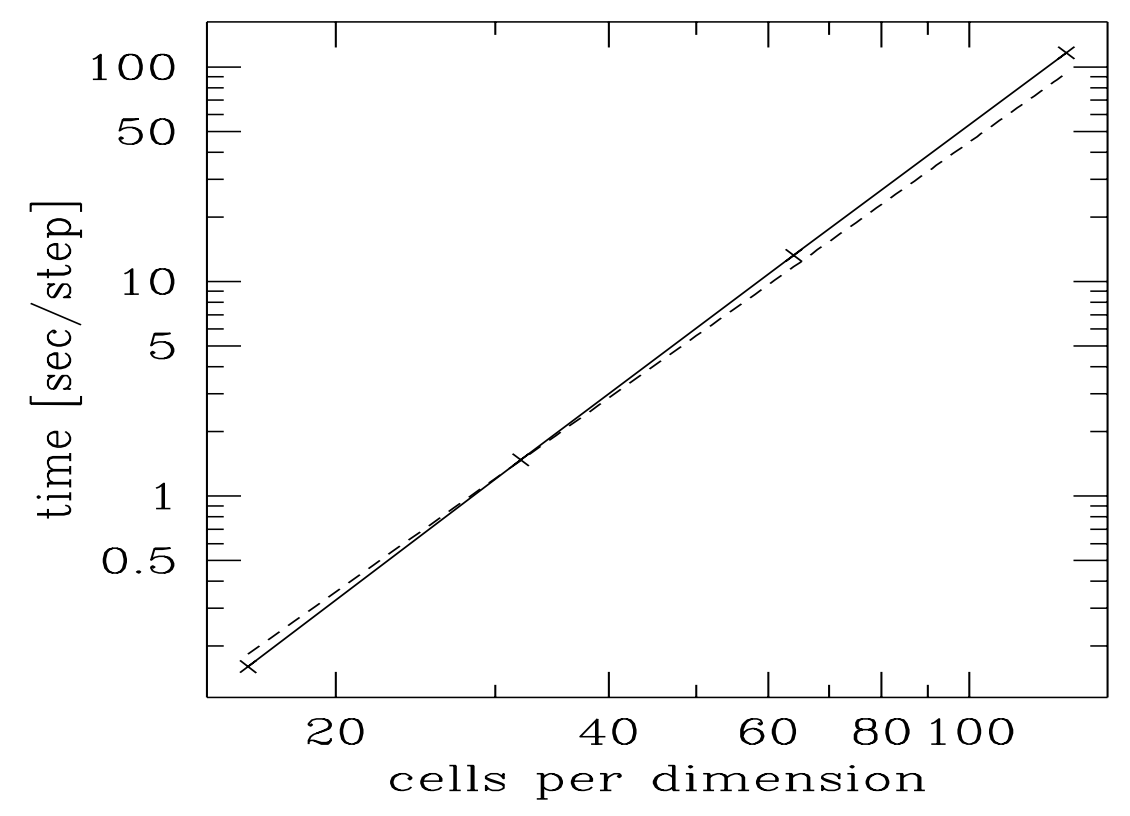

Fig. 14. CPU-time per step used by the FFT-routine for different grid-resolutions (solid line). The dashed line shows $t \propto N_{\mathrm{gc}}=N^{3}$. 


\section{Comparison with other Codes}

Demonstration of the conservation of energy and angular momentum is a necessary but not sufficient condition for validating any particle-mesh code. Additional validation of the code comes from an inter-comparison of results obtained with entirely different numerical techniques. Such a comparison is available in the study of the tidal dissolution of small satellite galaxies orbiting in an extended Galactic dark halo (Klessen \& Kroupa 1998). Such an application of SUPERBOX is rather extreme in that the jump in resolution from the middle grid $(0.29 \mathrm{kpc} / \mathrm{cell}$ length $)$ containing the small satellite to the outermost grid ( $25 \mathrm{kpc} / \mathrm{cell}$ length) containing the 'local universe' is very large.

In that study, two SUPERBOX computations are compared with calculations done using a direct but softened N-body integrator on the special hardware device named GRAPE 3 connected with a SUN-Ultra 20. The SUPERBOX calculation uses $N_{\mathrm{gc}}=$ $32^{3}$ grids, has $3 \times 10^{5}$ satellite particles, and treats the live Galactic dark halo with $10^{6}$ particles. The GRAPE computation uses $1.3 \times 10^{5}$ satellite particles with the Galactic dark halo being an analytic isothermal potential. Both calculations proceed for many thousands of time steps, corresponding to a time interval of many Gyr, until beyond disruption of the satellite. The results are in nice agreement, yielding essentially the same behaviour of the satellite, apart from expected (small) differences in exact disruption time. Of interest is also a comparison in CPU times required for the calculations, keeping in mind the very different number of particles used: with SUPERBOX it takes 0.57 days on an IBM RISC/6000 workstation to compute 1 Gyr using $1.3 \times 10^{6}$ particles, and it takes 0.36 days with GRAPE using $1.3 \times 10^{5}$ particles (with an $\mathcal{O}\left(N_{\mathrm{p}}^{2}\right)$ scaling).

In the above calculations, the satellite mass is too small for the orbit to be affected by dynamical friction. A comparison of the orbital decay through dynamical friction of a massive satellite is of interest with analytical estimates, because this allows an altogether independent verification if SUPERBOX treats collective behaviour on larger scales correctly. This is discussed in Section 7.

\section{Dynamical Friction}

An object moving through a homogeneous distribution of much lighter masses induces a trailing over-density. This over-density attracts the object which, as a result, is decelerated (Chandrasekhar 1943).

The orbital decay of a satellite galaxy or globular cluster orbiting within a larger galaxy is an astrophysical process relevant, for example, to the rate at which a satellite population is depleted and to the built-up of galactic bulges. In the Local Group, 
dynamical friction affects the orbit of the Magellanic Clouds, which is important for tracing their origin (see Westerlund 1997 and Kroupa \& Bastian 1997 for summaries). It may also change the orbit of the Sagittarius dwarf spheroidal galaxy (Ibata \& Lewis 1998; Gomez-Flechoso et al. 1999) and other nearby companions, depending on their masses. Understanding and proper application of dynamical friction is thus a very important issue.

The question if Chandrasekhar's formula (Eq. 30 below) can be applied to orbits within finite and inhomogeneous density distributions has been the issue of a significant debate throughout the 1970's and the 1980's. The result is that for satellite masses that are relatively small compared to the large galaxy, the formula is a good approximation (Bontekoe \& van Albada 1987; Zaritsky \& White 1988; Velazquez \& White 1999). Massive satellites induce non-local perturbations in the larger galaxy that are not taken account of in the derivation of Eq. 30, and in the collision of two galaxies of comparable mass analytical estimates become intractable, and the self-consistent numerical experiment must be resorted to (e.g. Madejski \& Bien 1993). In order to do this we must, however, have reason to trust the numerical results.

To demonstrate the reliability of our results, we compare the orbital evolution of a satellite galaxy orbiting in an extended dark halo computed with the fully selfconsistent SUPERBOX-code, with the evolution resulting from the by now wellestablished Chandrasekhar approximation (Eq. 30).

\subsection{Calculations with SUPERBOX}

For the numerical experiment a compact spherical satellite galaxy is injected into an extended isothermal dark parent halo. The numerical rendition of both systems is described in detail in Kroupa (1997), and only a short description is given here.

The parent halo is assumed to extend to $R_{\mathrm{c}}=250 \mathrm{kpc}$ with a core radius $\gamma=5 \mathrm{kpc}$ and a total mass $M_{\text {halo }}=2.85 \times 10^{12} M_{\odot}$, corresponding to a circular velocity $V_{\mathrm{c}}=220 \mathrm{~km} / \mathrm{s}$. A particle takes $588 \mathrm{Myr}$ to cross the 33 per cent mass diameter. The inner, middle and outer grids are centred on the density maximum and have edge lengths of 50, 188 and $700 \mathrm{kpc}$, respectively. The model is allowed to relax to virial equilibrium, after which state it has a slightly more compact configuration with a circular velocity at a radius of $150 \mathrm{kpc}$ of $245 \mathrm{~km} / \mathrm{s}$, corresponding to a mass within that radius of about $2.1 \times 10^{12} M_{\odot}$. Due to a mild radial orbit instability the halo is also slightly prolate.

Plummer density distributions are taken for the satellites, each with a Plummer radius $R_{\mathrm{pl}}=3 \mathrm{kpc}$ and a cutoff radius of $15 \mathrm{kpc}$. The innermost and middle grids are centred on the satellite's density maximum and have edge lengths of 10 and $40 \mathrm{kpc}$, respectively. The outer grid is the same as for the halo. Three satellite 
masses are used, $M_{\text {sat }}=1 \times 10^{10}, 7 \times 10^{10}$ and $5 \times 10^{11} M_{\odot}$. The respective crossing times of the 33 per cent mass diameter are $t_{\mathrm{cr}, 33}=84,32$ and $12 \mathrm{Myr}$.

For the calculation, $N=32$ grid cells per dimension are used (only 28 being active, see Section 2.3), with $10^{6}$ particles in the parent halo, and $3 \times 10^{5}$ satellite particles. For the halo the resolution is thus $1.79 \mathrm{kpc} /$ cell-length for $r \leq 25 \mathrm{kpc}, 6.71 \mathrm{kpc} / \mathrm{cell}-$ length for $25 \mathrm{kpc}<r \leq 94 \mathrm{kpc}$ and $25 \mathrm{kpc} /$ cell-length for $94 \mathrm{kpc}<r \leq 350 \mathrm{kpc}$. For the satellite the resolution is $0.36 \mathrm{kpc} / \mathrm{cell}-$ length for $r_{\mathrm{s}} \leq 5 \mathrm{kpc}, 1.43 \mathrm{kpc} / \mathrm{cell}-$ length for $5 \mathrm{kpc}<r_{\mathrm{s}} \leq 20 \mathrm{kpc}$ and $25 \mathrm{kpc} /$ cell-length for $20 \mathrm{kpc}<r_{\mathrm{s}} \leq 350 \mathrm{kpc}$, where $r_{\mathrm{s}}$ is the distance from the satellite's density maximum. The integration step size is $\Delta t=t_{\mathrm{cr}, 33} / 75$.

The satellite is allowed to relax to virial equilibrium before being placed into the parent halo at an initial radius $r(0)=|\mathbf{r}(t=0)|=150 \mathrm{kpc}$ with an initial velocity $v(0)=|\mathbf{v}(t=0)|=245 \mathrm{~km} / \mathrm{s}$ perpendicular to the radius vector, $\mathbf{r}$.

The satellite's galactocentric distance is $r(t)=\left|\mathbf{r}_{\text {sat }}(t)-\mathbf{r}_{\text {halo }}(t)\right|$, and its velocity is $v(t)=\left|\mathbf{v}_{\text {sat }}(t)-\mathbf{v}_{\text {halo }}(t)\right|$, where $\mathbf{r}_{\text {sat }}(t)$ and $\mathbf{r}_{\text {halo }}(t)$ are the position vectors of the density maxima of the satellite and halo, respectively, and $\mathbf{v}_{\text {sat }}(t)$ and $\mathbf{v}_{\text {halo }}(t)$ are the velocity vectors of the satellite's and halo's density maxima, respectively.

\subsection{Chandrasekhar friction}

The equation of motion of a satellite in a stationary and rigid isothermal parent halo is

$$
\begin{aligned}
\frac{\mathrm{d}^{2} \mathbf{r}}{\mathrm{d} t^{2}} & =a_{\mathrm{g}} \mathbf{r}+\eta \mathbf{v}, \\
a_{\mathrm{g}} & =-\frac{V_{\mathrm{c}}^{2}}{\gamma^{2}+r^{2}} \\
\eta & =-4 \pi G^{2} \ln \Lambda \frac{\rho(r) M_{\mathrm{sat}}}{v^{3}}\left[\operatorname{erf}(x)-\frac{2 x \mathrm{e}^{-x^{2}}}{\sqrt{\pi}}\right],
\end{aligned}
$$

where $a_{\mathrm{g}}$ is the acceleration in an isothermal halo, and $\eta$ is the deceleration due to dynamical friction, in which $G=4.499 \times 10^{-3} \mathrm{pc}^{3} /\left(M_{\odot} \mathrm{Myr}^{2}\right)$ is the gravitational constant, $\rho(r)=V_{\mathrm{c}}^{2} /\left[4 \pi \mathrm{G}\left(\gamma^{2}+r^{2}\right)\right]$ is the halo density and $\operatorname{erf}(x)$ is the error function with $x=v / V_{\mathrm{c}}$. A derivation of Chandrasekhar's dynamical friction formula can be found in Binney \& Tremaine (1987).

The numerical value of the Coulomb logarithm, $\ln \Lambda=b_{\max } / b_{\min }$, is somewhat illdefined. It is calculated by integrating particle deflections over impact parameters ranging from a minimum $\left(b_{\min }\right)$ to a maximum $\left(b_{\max }\right)$ effective value. The minimum impact parameter is taken here to be $b_{\min }=2.7 \mathrm{kpc}$. The maximum impact 
parameter, however, is something like the distance over which $\rho(r)$ falls off significantly, and is thus less-well defined. An upper limit is $b_{\max } \approx r(t=0)=150 \mathrm{kpc}$. It can also be estimated by assuming $\rho\left(r+b_{\max }^{+}\right)=0.5 \rho(r)$. With $r=150 \mathrm{kpc}$, $b_{\max }^{+}=62 \mathrm{kpc}$. If, on the other hand, $\rho\left(r-b_{\max }^{-}\right)=2 \rho(r)$, then with $r=150 \mathrm{kpc}$, $b_{\max }^{-}=44 \mathrm{kpc}$. Once the satellite orbit decays to $r=30 \mathrm{kpc}$, then $b_{\max }^{+}=13 \mathrm{kpc}$, while $b_{\max }^{-}=9 \mathrm{kpc}$. The Coulomb logarithm thus lies in the range $\ln \Lambda \approx 1$ to 4 , and the above argument suggests that it may be a monotonically decreasing function of declining $r(t)$.

Apart from the somewhat arbitrary value of the Coulomb logarithm, the analytic derivation of Eq. 32 assumes a Maxwellian velocity distribution in the halo, neglects the self gravity of the wake induced by the satellite's gravitational focusing, and the motion of the deflected halo particles is assumed to be governed only by the satellite - the gravitational field of the parent halo is neglected. Nevertheless, Eq. 32 has been shown to provide reliable results provided $M_{\text {sat }} / M_{\text {halo }} \lesssim 0.2$ and $\gamma<r(t)<R_{\mathrm{c}}$ (Binney \& Tremaine 1987 and references therein). The SUPERBOX model described in Section 7.1 conforms with these restrictions.

For the comparison with SUPERBox, $V_{\mathrm{c}}=245 \mathrm{~km} / \mathrm{s}, \gamma=3 \mathrm{kpc}$, and $M_{\mathrm{sat}}=$ $1 \times 10^{10}, 7 \times 10^{10}$ and $5 \times 10^{11} M_{\odot}$. Eq. 30 is rewritten to four coupled first-order differential equations, and integrated numerically using the Runga-Kutta method, with a sufficiently small step size to ensure stability of the solution.

\subsection{Results}

Since the Coulomb logarithm is ill defined, it is necessary to first calibrate the numerical solution to Eq. 30 using one SUPERBOX calculation. Fig. 15 shows the decay of the orbit of the satellite with $M_{\text {sat }}=7 \times 10^{10} M_{\odot}$ according to SUPERBOX and Eq. 30 under different assumptions for $\ln \Lambda$.

For the solution shown as the lower-dotted- $r(t)$ curve in Fig. $15, \ln \Lambda=4$. The SUPERBOX result requires a smaller Coulomb logarithm. Tests show that $\ln \Lambda=1.6$ leads to good agreement. To show a possible upper limit to the solution of the equation of motion, the case $\ln \Lambda=\left(0.049 r(t) / b_{\text {min }}\right)$ is plotted as the upper-dotted$r(t)$ curve. For this case, $\ln \Lambda=1$ initially, and it decreases with $r(t)$. The figure also shows the time-varying distance along the $\mathrm{x}$-axis between the density maxima of the satellite and the halo. The damped oscillation is nicely evident, and the period of the orbit is initially 3.2 Gyr. The lower panel of Fig. 15 plots the solutions for $v(t)$. The satellite in the SUPERBOX calculation retains an approximately constant velocity, as is expected from the theory of dynamical friction, the solutions from which are plotted with different lines corresponding to the cases discussed above.

The overall conclusion is that the SUPERBOX result is consistent with theoretical expectations, provided $\ln \Lambda=1$. 6 . That this finding extends to other satellite masses 
is shown in Fig. 16. In all cases, the satellites have lost less than 20 per cent of their mass by the end of the calculation.

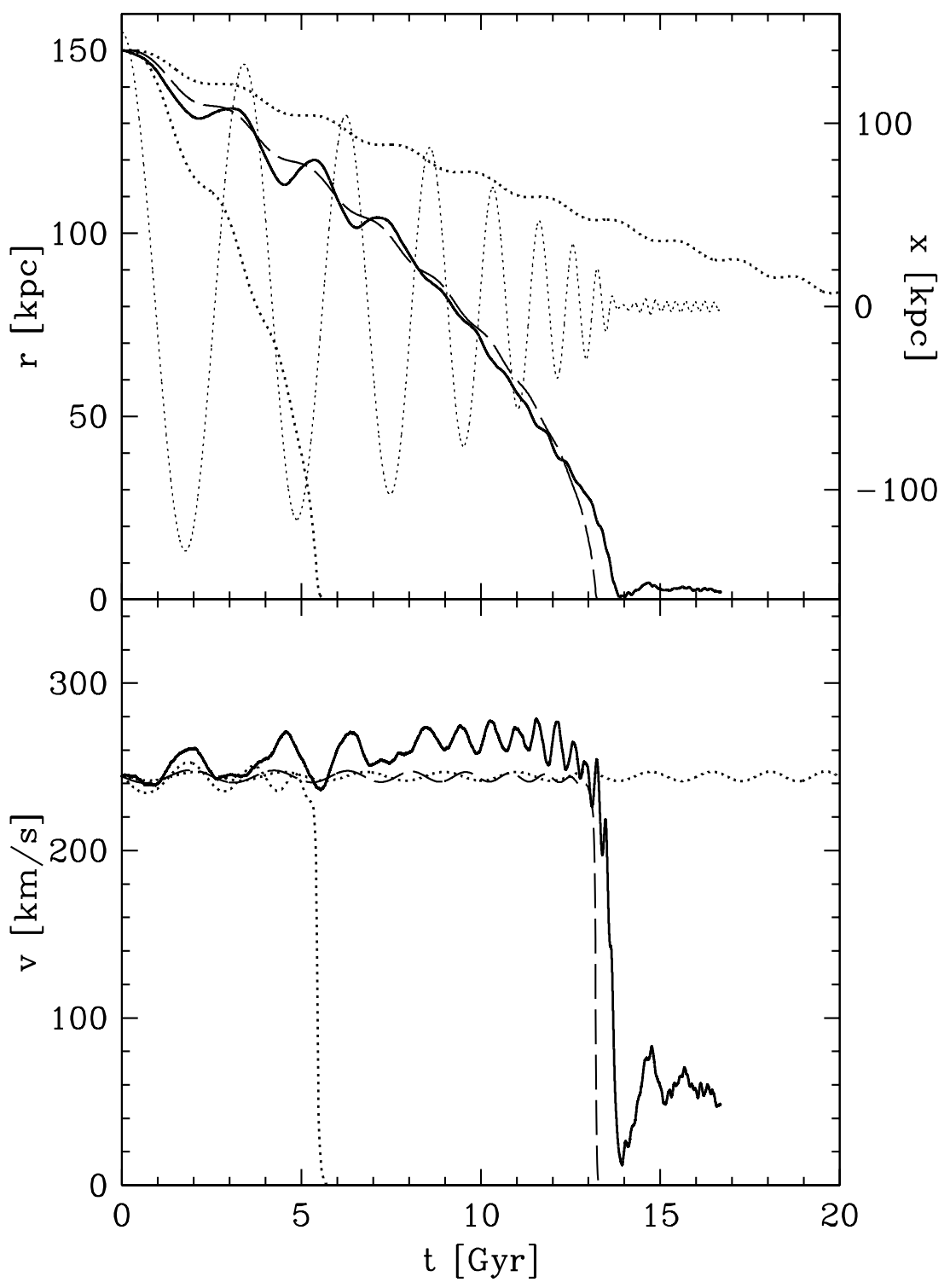

Fig. 15. The time evolution of the satellite's galactocentric distance, $r$ (upper panel), and velocity, $v$ (lower panel). The satellite has a mass $M_{\text {sat }}=7 \times 10^{10} M_{\odot}$ and orbits in an extended isothermal dark halo. The SUPERBOX result is shown as the thick solid curve. The other (theoretical) curves result from numerically integrating Eq. 30 assuming $\ln \Lambda=4$ (lower dotted curve in top panel), $\ln \Lambda=1.6$ (long-dashed curve) and $\ln \Lambda(t)=\ln \left(0.049 r(t) / b_{\min }\right)$ with $b_{\min }=2.7 \mathrm{kpc}$ (upper dotted curve in top panel). The SUPERBOX evolution of $x(t)=x_{\text {sat }}(t)-x_{\text {halo }}(t)$ is plotted as the thin dotted line in the upper panel. 


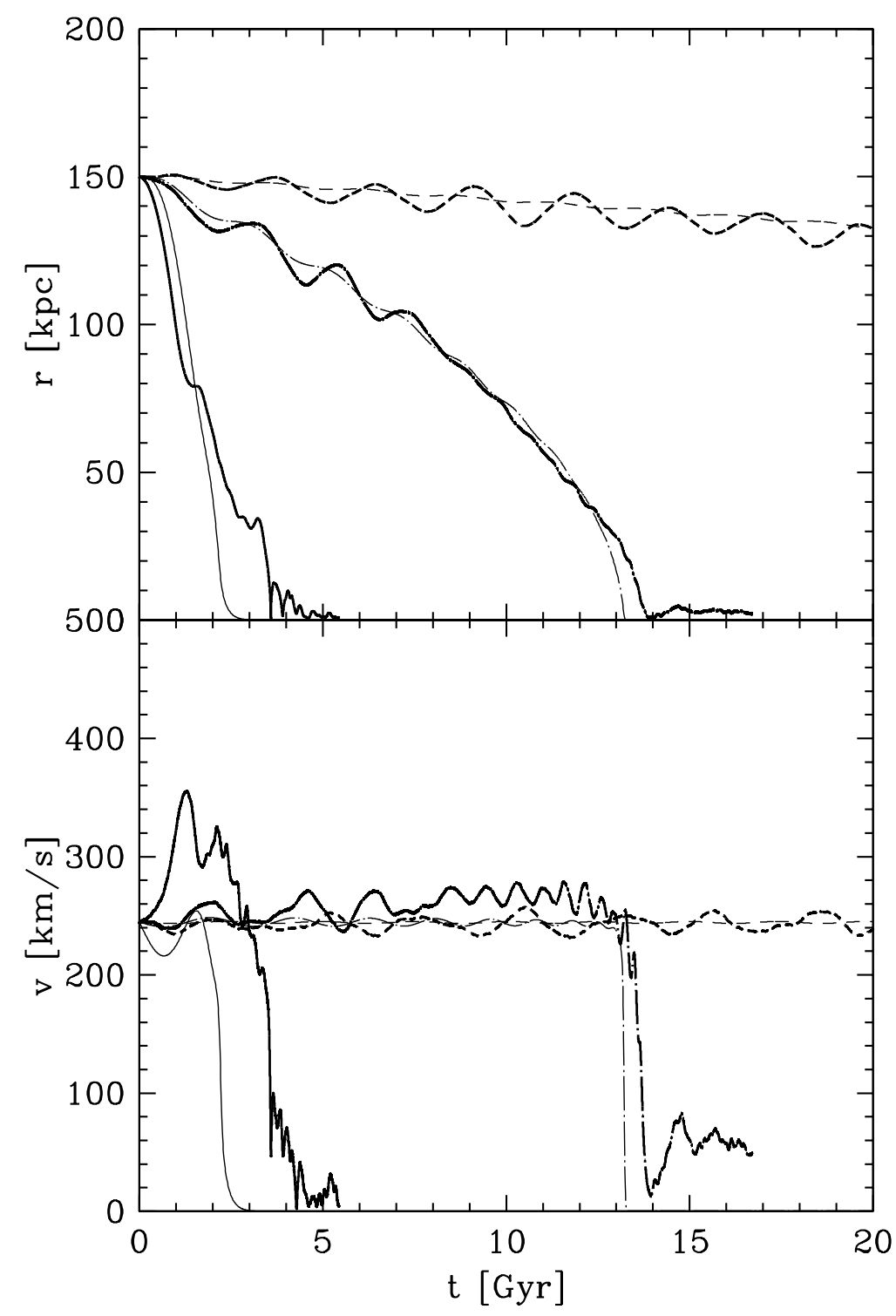

Fig. 16. The time evolution of the satellite's galactocentric distance, $r$ (upper panel), and velocity, $v$ (lower panel). The SUPERBOX satellite has a mass $M_{\text {sat }}=5 \times 10^{11}, M_{\odot}$ (solid curve), $7 \times 10^{10} M_{\odot}$ (dash-dotted curve) and $1 \times 10^{10} M_{\odot}$ (dashed curve), and orbits in an extended isothermal dark halo. The case discussed in Fig. 15 is identical to the present SUPERBOX case plotted as the dash-dotted curve. Eq. 30, with $\ln \Lambda=1.6$, produces the corresponding solutions shown as thin lines.

It is notable that one single value for the Coulomb logarithm applies to the whole radial range as well as to all three satellites, suggesting that non-local perturbations of the extended halo do not significantly alter the orbit of the satellites. This finding is in nice agreement with the results for $\ln \Lambda$ by Bontekoe \& van Albada (1987) and Velazquez \& White (1999), and support their finding that Chandrasekhar's approximation yields a useful description of orbital evolution if $\ln \Lambda \approx 1.5$ is used. 
Further calculations with more grid cells and a more detailed study will be needed to show if the oscillations in $r(t)$ and $v(t)$ seen in Figs. 15 and 16, as well as in similar figures in the literature (e.g. fig. 3 in Bontekoe \& van Albada 1987) are physical, i.e. if they are manifestations of the dependency of $\eta$ (Eq. 32) on $r$ and $v$.

\section{Conclusions}

SUPERBOX is an unconventional particle-mesh code that uses moving sub-grids to track and resolve high-density peaks in the particle distribution. This extension avoids the limitation in spatial resolution encountered with standard particle mesh codes that employ only a single grid. The code is efficient in that the computational overhead is kept as slim as possible. The code is also memory efficient by using only one set of grids to treat galaxies in succession.

In this paper we have explained the algorithms used for force evaluation, grid positioning and orbit integration. The nearest-grid-point scheme (NGP) is used to compute the density on a grid. The potential is obtained by FFT, from which accelerations at the position of each particle within a cell are calculated using second-order central-difference quotients with linear interpolation to the position of particles inside that cell. Such a scheme has been identified as a special higher-order NGP scheme and is competitive with the cloud-in-cell (CIC) scheme regarding the precision and continuity of the forces across grid boundaries. Together with the nested sub-grids, this leads to precise and reliable force calculations at the location of each particle. The sub-grids are positioned at each time-step on the density maximum or at the centre of mass of a galaxy, the former method being the more useful. For orbit integration the leap-frog scheme is used. Conservation of energy and angular momentum is excellent, and numerical relaxation is significantly longer than a Hubble time for a galaxy with a crossing time of about $10^{8} \mathrm{yr}$ and particle numbers exceeding $10^{5}$. CPU-timing and memory consumption are such that calculations of galaxy encounters involving many $\times 10^{6}$ particles are possible on present-day desk-top computers with 20MB RAM or more (see Fig. 12). On the other hand, it is clear that using the resources of massively parallel supercomputers will significantly increase the capabilities of SUPERBOX.

The most serious limitation of SUPERBOX lies in the inability to resolve newly formed self-gravitating systems, because the grid structure is decided on at the beginning of a computation. SUPERBOX has presently no scheme to allow the inclusion of additional grids during a calculation. Such objects can form in tidal arms and may be the precursors of some dwarf satellite galaxies. This limitation will be reduced as the number of grids that can be used increases owing to advances in computer technology.

Current and future developments of SUPERBOX would include for example incor- 
porating an individual time-step scheme per grid and galaxy. We intend to parallelise the code to run on CRAY T3E super-computers. Furthermore, additional grid-levels will be introduced to reduce the jump in resolution between the present middle and outer grids, and/or to further increase the resolution of the central regions of a galaxy. Finally, the current sticky-particle algorithm of SUPERBOX can be extented (e.g. to include star formation).

Problems that are now being tackled with SUPERBOX include computations of the tidal interactions of satellite and disk galaxies (J.M. Penarrubia, in preparation), and the possible formation of spheroidal satellite galaxies from stellar super clusters (Fellhauer et al.). Fellhauer \& Kroupa (2000) report on a study of the dynamical evolution of clusters of dozens to hundreds of young massive and compact star clusters (i.e. stellar super clusters) in a tidal field, in an attempt to understand the evolution of such objects observed in HST images of the Antennae galaxies (see e.g. Lançon et al. 2000). Also, the parameter survey of dwarf galaxies orbiting in a massive dark halo is being completed (Kroupa, in preparation), to identify regions in parameter space that may lead to $\mathrm{dSph}$-like systems without dark matter. Furthermore, collapse calculations are being performed to study the properties of the resulting object after violent relaxation and secular relaxation thereafter (Boily, in preparation).

\section{Acknowledgements}

P.K. and H.B. acknowledge financial support by SFB 328 and C.B, by SFB439 (at the University of Heidelberg) from the German Science Foundation (DFG). Support and advice on computational aspects of HLRS Stuttgart is gratefully acknowledged.

SUPERBOX is available at

ftp: \\ftp.ari.uni-heidelberg.de/pub/mike/super.tar.gz .

\section{References}

[1] Barnes J.E., Hut P., 1986, Nature, 324, 446

[2] Bien R., Fuchs B., Wielen R., 1991, in The CP90 Europhysics Conference on Computational Physics, Serie A, 228, 3

[3] Binney J., Tremaine S., 1987, Galactic Dynamics, Princeton University Press, New Jersey

[4] Bontekoe Tj.R., van Albada T.S., 1987, MNRAS, 224, 349

[5] Chandrasekhar S., 1943, ApJ, 97, 255

[6] Couchman H.M.P., 1999, to appear in Riffert H., Werner K. (eds.), Computational Astrophysics, The Journal of Computational and Applied Mathematics (JCAM), Elsevier Press, Amsterdam 
[7] Davé R., Dubinski J., Hernquist L., 1997, NewA, 2, 277

[8] Eastwood J.W., Brownrigg D.R.K., 1978, J. Comput. Phys., 32, 24

[9] Fellhauer M., 1996, diploma thesis, Univ. of Heidelberg

[10] Fellhauer M., Kroupa P., 2000, in ASP Conf. Series Vol. 211, Massive Stellar Clusters, ed. A. Lançon \& C.M. Boily (San Francisco: PASP), 241

[11] Gomez-Flechoso M.A., Fux R., Martinet L., 1999, A\&A, 347, 77

[12] Hockney R.W., Eastwood J.W., 1981, Computer Simulations Using Particles, McGraw-Hill

[13] Hohl F., 1970, NASA Technical Report R-343

[14] Ibata R.A., Lewis G.F., 1998, ApJ, 500, 575

[15] Klessen R., Kroupa P., 1998, ApJ, 498, 143

[16] Kravtsov A.V., Klypin A.A., Khokhlov A.M., 1997, ApJS, 111, 73

[17] Kroupa P., 1997, NewA, 2, 139

[18] Kroupa P., Bastian U., 1997, NewA, 2, 77

[19] Lançon A., Boily C.M. (eds.), 2000, ASP Conf. Series Vol. 211, Massive Star Clusters, San Francisco: PASP, pp. 330

[20] Madejski R., Bien R., 1993, A\&A, 280, 383

[21] Pearce F.R., Couchman H.M.P., 1997, NewA, 2, 411

[22] Press W.H., Teukolsky S.A., Vetterling W.T., Flannery B.P., 1986, Numerical Recipes in Fortran, Cambridge University Press, Cambridge

[23] Sellwood J.A., 1987, ARA\&A, 25, 151

[24] Spitzer L., Hart M.H., 1971, ApJ, 164, 399

[25] Spurzem R., 1999, Journal of Computational and Applied Mathematics, 110, Elsevier, in press

[26] Standish E.M., Aksnes K., 1969, ApJ, 158, 519

[27] Velazquez H., White S.D.M., 1999, MNRAS, 304, 254

[28] Villumsen J.V., 1989, ApJS, 71, 407

[29] Wassmer N., 1992, diploma thesis, Univ. of Heidelberg

[30] Wassmer N., Bien R., Fuchs B., Wielen R., 1993, Astron. Ges. Abstr. Ser., 9, 78

[31] Westerlund B.E., 1997, The Magellanic Clouds, Cambridge University Press, Cambridge

[32] Werner H., Schabach R., 1979, Praktische Mathematik II, Springer Verlag

[33] Zaritsky D., White S.D.M., 1988, MNRAS, 235, 289 